PHYSICAL REVIEW D 89, 042002 (2014)

\title{
Template banks for binary black hole searches with numerical relativity waveforms
}

\author{
Prayush Kumar, ${ }^{1, *}$ Ilana MacDonald, ${ }^{2,3}$ Duncan A. Brown, ${ }^{1,4}$ Harald P. Pfeiffer, ${ }^{2,5}$ Kipp Cannon, ${ }^{2}$ Michael Boyle, ${ }^{6}$ \\ Lawrence E. Kidder, ${ }^{6}$ Abdul H. Mroué, ${ }^{2}$ Mark A. Scheel, ${ }^{7}$ Béla Szilágyi, ${ }^{7}$ and Anıl Zenginoğlu ${ }^{7}$ \\ ${ }^{1}$ Department of Physics, Syracuse University, Syracuse, New York 13244, USA \\ ${ }^{2}$ Canadian Institute for Theoretical Astrophysics, University of Toronto, \\ Toronto, Ontario M5S 3H8, Canada \\ ${ }^{3}$ Department of Astronomy and Astrophysics, University of Toronto, Toronto, Ontario M5S 3H4, Canada \\ ${ }^{4}$ LIGO Laboratory, California Institute of Technology, Pasadena, California 91125, USA \\ ${ }^{5}$ Canadian Institute for Advanced Research, 180 Dundas St. West, Toronto, Ontario M5G 1Z8, Canada \\ ${ }^{6}$ Center for Radiophysics and Space Research, Cornell University, Ithaca, New York 14853, USA \\ ${ }^{7}$ Theoretical Astrophysics 350-17, California Institute of Technology, Pasadena, California 91125, USA
}

(Received 13 November 2013; published 18 February 2014)

\begin{abstract}
Gravitational waves from coalescing stellar-mass black hole binaries (BBHs) are expected to be detected by the Advanced Laser Interferometer gravitational-wave observatory and Advanced Virgo. Detection searches operate by matched filtering the detector data using a bank of waveform templates. Traditionally, template banks for BBHs are constructed from intermediary analytical waveform models which are calibrated against numerical relativity simulations and which can be evaluated for any choice of BBH parameters. This paper explores an alternative to the traditional approach, namely, the construction of template banks directly from numerical BBH simulations. Using nonspinning BBH systems as an example, we demonstrate which regions of the mass-parameter plane can be covered with existing numerical $\mathrm{BBH}$ waveforms. We estimate the required number and required length of BBH simulations to cover the entire nonspinning BBH parameter plane up to mass ratio 10, thus illustrating that our approach can be used to guide parameter placement of future numerical simulations. We derive error bounds which are independent of analytical waveform models; therefore, our formalism can be used to independently test the accuracy of such waveform models. The resulting template banks are suitable for advanced LIGO searches.
\end{abstract}

DOI: 10.1103/PhysRevD.89.042002

PACS numbers: 04.80.Nn, 95.55.Ym, 04.25.Nx, 04.25.dg

\section{INTRODUCTION}

Upgrades to the LIGO and Virgo observatories are underway [1,2], with first observation runs planned for 2015 [3]. The construction of the Japanese detector KAGRA has also begun [4]. The advanced detectors will be sensitive to gravitational waves at frequencies down to $\sim 10 \mathrm{~Hz}$, with an order of magnitude increase in sensitivity across the band. This is a significant improvement over the lower cutoff of $40 \mathrm{~Hz}$ for initial LIGO. Estimates for the expected rate of detection have been placed between 0.4 and 1000 stellar-mass binary black hole (BBH) mergers a year [5]. The uncertainty in these estimates comes from the uncertainties in the various factors that govern the physical processes in the BBH formation channels [6,7]. In subsolar metallicity environments, stars (in binaries) are expected to lose relatively less mass to stellar winds and form more massive remnants [8-10]. Population synthesis studies estimate that subsolar metallicity environments within the horizon of advanced detectors could increase the detection rates to be as high as a few thousand per year $[11,12]$. On the other hand, high recoil momenta during

*prayush.kumar@ligo.org core collapse, and merger during the common-envelope phase of the binary star evolution could also decrease the detection rates drastically $[10,11]$.

Past gravitational wave (GW) searches have focused on GW bursts [13-15], coalescing compact binaries [16-22], and ringdowns of perturbed black holes [23], among others [24-28]. For coalescing BBHs, detection searches involve matched filtering $[29,30]$ of the instrument data using large banks of theoretically modeled waveform templates as filters [31-36]. The matched filter is the optimal linear filter to maximize the signal-to-noise ratio (SNR), in the presence of stochastic noise [37]. It requires an accurate modeling of the gravitational waveform emitted by the source binary. Early LIGO-Virgo searches employed template banks of post-Newtonian (PN) inspiral waveforms [16-20], while more recent searches targeting high-mass BBHs used complete inspiral-merger-ringdown waveform templates [21,22].

Recent developments in numerical relativity (NR) have provided complete simulations of $\mathrm{BBH}$ dynamics in the strong-field regime, i.e., during the late-inspiral and merger phases [38-42]. These simulations have contributed unprecedented physical insights to the understanding of 
BBH mergers (see, e.g., Refs. [43-46] for recent overviews of the field). Because of their high computational cost, fully numerical simulations currently span a few tens of inspiral orbits before merger. For mass ratios $q=m_{1} / m_{2}=1,2,3$, 4, 6, 8, the multidomain Spectral Einstein code (SpEC) [47] has been used to simulate 15-33 inspiral and merger orbits [48-50]. These simulations have been used to calibrate waveform models, for example, within the effective-onebody (EOB) formalism [51-54]. Alternately, inspiral waveforms from PN theory can be joined to numerical $\mathrm{BBH}$ inspiral and merger waveforms, to construct longer hybrid waveforms [55-59]. NR-PN hybrids have been used to calibrate phenomenological waveform models $[60,61]$, and within the NINJA project $[62,63]$, they have been used to study the efficacy of various GW search algorithms toward realistic (NR) signals $[64,65]$.

Constructing template banks for gravitational wave searches has been a long sought goal for NR. Traditionally, intermediary waveform models are calibrated against numerical simulations and then used in template banks for LIGO searches [21,22]. In this paper we explore an alternative to this traditional approach, proposing the use of NR waveforms themselves and hybrids constructed out of them as search templates. For a proof of principle, we focus on the nonspinning $\mathrm{BBH}$ space, with the aim of extending to spinning binaries in future work. We investigate exactly where in the mass space the existing NR waveforms/hybrids can be used as templates, finding that only six simulations are sufficient to cover binaries with $m_{1,2} \gtrsim 12 M_{\odot}$ up to mass ratio 10 . This method can also be used as a guide for the placement of parameters for future NR simulations. Recent work has shown that existing PN waveforms are sufficient for aLIGO searches for $M=m_{1}+m_{2} \lesssim 12 M_{\odot}[66,67]$. To extend the NR/hybrid bank coverage down to $M \simeq 12 M_{\odot}$, we demonstrate that a total of 26 simulations would be sufficient. The template banks are constructed with the requirement that the net SNR recovered for any BBH signal should remain above $96.5 \%$ of its optimal value. Enforcing this tells that that these 26 simulations would be required to be $\sim 50$ orbits long. This goal is achievable, given the recent progress in simulation technology $[50,57,68]$. Our template banks are viable for GW searches with aLIGO, and the framework for using hybrids within the LIGO-Virgo software framework has been demonstrated in the NINJA-2 collaboration [69]. In this paper, we also derive waveform modeling error bounds which are independent of analytical models. These can be extended straightforwardly to assess the accuracy of such models.

First, we construct a bank for purely NR templates, restricting to currently available simulations [48-50,57]. We use a stochastic algorithm similar to Refs. [70-72] and place a template bank grid constrained to $q=m_{1} / m_{2}=$ $\{1,2,3,4,6,8\}$. The bank placement algorithm uses the EOB model from Ref. [53] (EOBNRv2). As this model was calibrated against NR for most of these mass ratios, we expect the manifold of EOBNRv2 to be a reasonable approximation for the NR manifold. In Sec. V, we demonstrate that this approximation holds well for NRPN hybrids as well. To demonstrate the efficacy of the bank, we measure its fitting factors (FFs) [73] over the BBH mass space. We simulate a population of 100,000 BBH waveforms with masses sampled uniformly over $3 M_{\odot} \leq m_{1,2} \leq 200 M_{\odot}$ and $M=m_{1}+m_{2} \leq 200 M_{\odot}$ and filter them through the template bank to characterize its SNR recovery. For a bank of NR templates, any SNR loss accrued will be due to the coarseness of the bank grid. We measure this requiring both signals and templates to be in the same manifold, using the EOBNRv2 model for both. We find that for systems with chirp mass $\mathcal{M}_{c} \equiv\left(m_{1}+\right.$ $\left.m_{2}\right)^{-1 / 5}\left(m_{1} m_{2}\right)^{3 / 5}$ above $\sim 27 M_{\odot}$ and $1 \leq q \leq 10$, this bank has FFs $\geq 97 \%$ and is sufficiently accurate to be used in GW searches. We also show that the coverage of the purely NR bank can be extended to include $10 \leq$ $q \leq 11$ if we instead constrain it to templates with mass ratios $q=\{1,2,3,4,6,9.2\}$.

Second, we demonstrate that currently available NR-PN hybrid waveforms can be used as templates to search for BBHs with much lower masses. The hybrids used correspond to mass ratios $q=\{1,2,3,4,6,8\}$. We use two distinct methods of bank placement to construct a bank with these mass ratios and compare the two. The first method is the stochastic algorithm we use for purely NR templates. The second is a deterministic algorithm, which constructs the two-dimensional bank (in $M$ and $q$ ) through a union of six one-dimensional banks, placed separately for each allowed value of mass ratio. Templates are placed over the total mass dimension by requiring that all pairs of neighboring templates have the same noise weighted overlap. As before, we measure the SNR loss from both banks, due to the discrete placement of the templates, by simulating a population of $100,000 \mathrm{BBH}$ signals, to find the SNR recovered. We measure the intrinsic hybrid errors using the method of Refs. [56,57] and subsequently account for them in the SNR recovery fraction. We find that the NR-PN hybrid bank is effectual for detecting BBHs with $m_{1,2} \geq 12 M_{\odot}$, with FFs $\geq 96.5 \%$. The number of templates required was found to be close to that of a bank constructed using the second-order TaylorF2 hexagonal bank placement algorithm [31-36]. We note that by pregenerating the template for the least massive binary for each of the mass ratios that contribute to the bank, we can rescale it on the fly to different total masses in the frequency domain [74]. Used in detection searches, such a bank would be computationally inexpensive to generate relative to a bank of time-domain modeled waveforms.

Finally, we determine the minimal set of NR simulations that we would need to extend the bank down to $M \simeq 12 M_{\odot}$. We find that a bank that samples from the set of 26 mass ratios listed in Table II would be sufficiently 
dense, even at the lowest masses, for binaries with mass ratios $1 \leq q \leq 10$. We show that this bank recovers more than $98 \%$ of the optimal SNR, not accounting for hybrid errors. To restrict the loss in the event detection rate below $10 \%$, we restrict the total SNR loss below 3.5\%. This implies the hybrid error mismatches stay below $1.5 \%$, which constrains the length of the NR part for each hybrid. We find that NR simulations spanning about 50 orbits of late inspiral, merger, and ringdown would suffice to reduce the PN truncation error to the desired level. With such a bank of NR-PN hybrids and purely PN templates for lower masses, we can construct GW searches for stellar-mass BBHs with mass ratios $q \leq 10$.

The paper is organized as follows, in Sec. II A, we discuss the NR waveforms used in this study; in Sec. II B, we describe the PN models used to construct the NR-PN hybrids; and in Sec. II C, we describe the construction of hybrid waveforms. In Sec. II D, we describe the EOB model that we use to place and test the template banks. In Sec. III, we describe the accuracy measures used in quantifying the loss in the signal-to-noise ratio in a matched-filtering search when using a discrete bank of templates and in the construction of hybrid waveforms. In Sec. IV, we describe the construction and efficacy of the NR-only banks, while in Sec. V, we discuss the same for the NR-PN hybrid template banks constructed with currently available NR waveforms. In Sec. VI, we investigate the parameter and length requirements for future NR simulations in order to cover the entire nonspinning parameter space with $12 M_{\odot} \leq M \leq 200 M_{\odot}, \quad m_{1,2} \geq 3 M_{\odot}$, and $1 \leq q \leq 10$. Finally, in Sec. VII, we summarize the results.

\section{WAVEFORMS}

In the sections that follow, we will describe the construction of template banks for NR or NR-PN hybrid waveform templates. The NR waveforms that we use correspond to mass ratios $q=\{1,2,3,4,6,8\}$ and were simulated using the SpEC code [47]. The construction of hybrid waveforms involves joining a long inspiral portion, modeled using PN theory, to the merger-ringdown waveform from NR. In this section, we describe both the NR waveforms and the PN models used in our study. Measuring the effectualness of these banks involves simulating a population of $\mathrm{BBH}$ signals. We use the recently published EOBNRv2 model [53] to obtain waveforms for BBHs with arbitrary masses. This model was calibrated against five out of the six NR simulations we use to construct our banks and is expected to be faithful at comparable mass ratios [53]. In this section, we briefly summarize the construction of EOBNRv2 waveforms as well.

\section{A. Numerical relativity simulations}

The numerical relativity waveforms used in this paper were produced with the SpEC code [47], a multidomain
TABLE I. SpEC BBH simulations used in this study. Given are symmetric mass ratio $\eta$, mass ratio $q=m_{1} / m_{2}$, and the length in orbits of the simulation. The last column gives the lowest total masses for which the NR simulations cover the entire coalescence process within the sensitive band of aLIGO, starting at $15 \mathrm{~Hz}$.

\begin{tabular}{lccc}
\hline \hline$\eta$ & & $\begin{array}{c}\text { Length } \\
\text { (in orbits) }\end{array}$ & $\begin{array}{c}\text { Minimum total } \\
\text { mass }\left(M_{\odot}\right)\end{array}$ \\
\hline 0.25 & $\mathrm{q}$ & 33 & 49 \\
0.2222 & 1 & 15 & 76 \\
0.1875 & 2 & 18 & 82 \\
0.1600 & 3 & 15 & 87 \\
0.1224 & 4 & 20 & 83 \\
0.0988 & 6 & 25 & 83 \\
\hline \hline
\end{tabular}

pseudospectral code to solve Einstein's equations. SpEC uses generalized harmonic coordinates, spectral methods, and a flexible domain decomposition, all of which contribute to it being one of the most accurate and efficient codes for computing the gravitational waves from binary black hole systems. High accuracy numerical simulations of the late inspiral, merger, and ringdown of coalescing binary black holes have been recently performed for mass ratios $q \equiv m_{1} / m_{2} \in\{1,2,3,4,6,8\}[48,49,75,76]$.

The equal-mass, nonspinning waveform covers 33 inspiral orbits and was first discussed in Refs. [49,57]. This waveform was obtained with numerical techniques similar to those of Ref. [48]. The unequal-mass waveforms of mass ratios 2, 3, 4, and 6 were presented in detail in Ref. [48]. The simulation with mass ratio 6 covers about 20 orbits, and the simulations with mass ratios 2, 3, and 4 are somewhat shorter and cover about 15 orbits. The unequal mass waveform with mass ratio 8 was presented as part of the large waveform catalog in Refs. [49,50]. It is approximately 25 orbits in length. We summarize the NR simulations used in this study in Table I. In the table, we also give the lowest total masses for which the NR waveforms span the aLIGO band, starting at $15 \mathrm{~Hz}$.

\section{B. Post-Newtonian waveforms}

PN theory is a perturbative approach to describing the motion of a compact object binary, during the slow-motion and weak-field regime, i.e., the inspiral phase. The conserved energy of a binary in orbit, $E$, has been calculated to 3PN order in literature [77-83]. Using the adiabatic approximation, we treat the course of inspiral as a series of radially shrinking circular orbits. This is valid during the inspiral when the angular velocity of the binary evolves more slowly than the orbital time scale. The radial separation shrinks as the binary loses energy to gravitational radiation that propagates outward from the system. The energy flux from a binary $F$ is known in PN theory to 3.5PN order [84-87]. Combining the energy balance equation, $\mathrm{d} E / \mathrm{d} t=-F$, with Kepler's law gives a description of the radial and orbital phase evolution of the binary. 
We start the waveform where the GW frequency enters the sensitive frequency band of advanced LIGO, i.e., at $15 \mathrm{~Hz}$. Depending on the way the expressions for orbital energy and flux are combined to obtain the coordinate evolution for the binary, we get different Taylor $\{\mathrm{T} 1, \mathrm{~T} 2, \mathrm{~T} 3, \mathrm{~T} 4\}$ time-domain approximants. Using the stationary phase approximation [88], frequency-domain equivalents of these approximants, i.e., TaylorFn, can be constructed. Past GW searches have extensively used the TaylorF2 approximant, as it has a closed form and mitigates the computational cost of generating and numerically Fourier transforming the time-domain template [16-20]. We refer the reader to Refs. [89,90] for an overview. From the coordinate evolution, we obtain the emitted gravitational waveform, approximating it by the quadrupolar multipole $h_{2, \pm 2}$, which is the dominant mode of the waveform.

\section{PN-NR hybrid waveforms}

The hybridization procedure used for this investigation is described in Sec. 3.3 of Ref. [56]: The PN waveform, $h_{\mathrm{PN}}(t)$, is time and phase shifted to match the NR waveform, $h_{\mathrm{NR}}(t)$, and they are smoothly joined together in a GW frequency interval centered at $\omega_{m}$ with width $\delta \omega$ :

$$
\omega_{m}-\frac{\delta \omega}{2} \leq \omega \leq \omega_{m}+\frac{\delta \omega}{2} .
$$

This translates into a matching interval $t_{\min }<t<t_{\max }$ because the GW frequency continuously increases during the inspiral of the binary. As argued in Ref. [56], we choose $\delta \omega=0.1 \omega_{m}$ because it offers a good compromise of suppressing residual oscillations in the matching time while still allowing $h_{\mathrm{PN}}(t)$ to be matched as closely as possible to the beginning of $h_{\mathrm{NR}}(t)$.

The PN waveform depends on a (formal) coalescence time, $t_{c}$, and phase, $\Phi_{c}$. These two parameters are determined by minimizing the GW phase difference in the matching interval $\left[t_{\min }, t_{\max }\right]$ as follows:

$$
t_{c}^{\prime}, \Phi_{c}^{\prime}=\underset{\mathrm{t}_{c}, \Phi_{c}}{\operatorname{argmin}} \int_{t_{\min }}^{t_{\max }}\left(\varphi_{\mathrm{PN}}\left(t ; t_{c}, \Phi_{c}\right)-\varphi_{\mathrm{NR}}(t)\right)^{2} \mathrm{~d} t,
$$

where $t_{c}^{\prime}$ and $\Phi_{c}^{\prime}$ are the time and phase parameters for the best matching between $h_{\mathrm{PN}}(t)$ and $h_{\mathrm{NR}}(t)$ and $\phi(t)$ is the phase of the $(2,2)$ mode of the gravitational radiation. Since we consider only the $(2,2)$ mode, this procedure is identical to time and phase shifting the PN waveform until it has the best agreement with NR as measured by the integral in Eq. (2). The hybrid waveform is then constructed in the form

$$
h_{\mathrm{H}}(t) \equiv \mathcal{F}(t) h_{\mathrm{PN}}\left(t ; t_{c}^{\prime}, \Phi_{c}^{\prime}\right)+[1-\mathcal{F}(t)] h_{\mathrm{NR}}(t),
$$

where $\mathcal{F}(t)$ is a blending function defined as

$$
\mathcal{F}(t) \equiv \begin{cases}1, & t<t_{\min } \\ \cos ^{2} \frac{\pi\left(t-t_{\min }\right)}{2\left(t_{\max }-t_{\min }\right)}, & t_{\min } \leq t<t_{\max } \\ 0 . & t \geq t_{\max } .\end{cases}
$$

In this work, we construct all hybrids using the same procedure, Eqs. (1)-(4), and vary only the PN approximant and the matching frequency $\omega_{m}$.

\section{Effective-one-body model}

Full numerical simulations are available for a limited number of binary mass combinations. We use a recently proposed EOB model [53], which we refer to as EOBNRv2, as a substitute to model the signal from binaries with arbitrary component masses in this paper. This model was calibrated to most of the numerical simulations that we use to construct templates banks, which span the range of masses we consider here well. So we expect this approximation to hold. We describe the model briefly here.

The EOB formalism maps the dynamics of a two-body system onto an effective mass moving in a deformed Schwarzschild-like background [51]. The formalism has evolved to use Padé resummations of perturbative expansions calculated from PN theory and allows for the introduction of higher (unknown) order PN terms that are subsequently calibrated against NR simulations of BBHs [52,91-94]. The EOBNRv2 model, that we use in this paper, has been calibrated to SpEC NR waveforms for binaries of mass ratios $q=\{1,2,3,4,6\}$.

The dynamics of the binary enters in the metric coefficient of the deformed Schwarzschild-like background, the EOB Hamiltonian [51], and the radiation-reaction force. These are known to 3PN order $[51,95]$ from PN theory. The $4 \mathrm{PN}$ and 5PN terms were introduced in the potential $A(r)$, which was Padé resummed and calibrated to NR simulations $[52,53,93,96,97]$. We use the resummed potential calibrated in Ref. [53] [see Eqs. (5)-(9)]. The geodesic dynamics of the reduced mass $\mu=m_{1} m_{2} / M$ in the deformed background is described by the Hamiltonian $H^{\text {eff }}$ given by Eq. (3) in Ref. [53]. The Hamiltonian describing the conservative dynamics of the binary (labeled the real Hamiltonian $H^{\text {real }}$ ) is related to $\hat{H}^{\text {eff }}$ as in Eq. (4) of Ref. [53]. The inspiral-merger dynamics can be obtained by numerically solving the Hamiltonian equations of motion for $H^{\text {real }}$; see, e.g., Eq. (10) of Ref. [53].

The angular momentum carried away from the binary by the outward propagating GWs results in a radiationreaction force that causes the orbits to shrink. This is due to the flux of energy from the binary, which is obtained by summing over the contribution from each term in the multipolar decomposition of the inspiral-merger EOB waveform. Complete resummed expressions for these multipoles [94] can be read off from Eqs. (13)-(20) of 
Ref. [53]. In this paper, as for PN waveforms, we model the inspiral-merger part by summing over the dominant $h_{2, \pm 2}$ multipoles.

The EOB ringdown portion is modeled as a sum of $N$ quasinormal modes, where $N=8$ for EOBNRv2 [52,96-98]. The ringdown frequencies depend on the mass and spin of the $\mathrm{BH}$ that is formed from the coalescence of the binary. The inspiral-merger and ringdown parts are attached by matching them at the time at which the amplitude of the inspiral-merger waveform peaks. [52,53]. The matching procedure followed is explained in detail Sec. II C of Ref. [53]. By combining them, we obtain the complete waveform for a BBH system.

\section{QUANTIFYING WAVEFORM ACCURACY AND BANK EFFECTUALNESS}

To assess the recovery of SNR from template banks with NR waveforms or NR-PN hybrids as templates, we use the measures proposed in Refs. [31,73,99]. The gravitational waveform emitted during and driving a $\mathrm{BBH}$ coalescence is denoted as $h(t)$, or simply $h$. The inner product between two waveforms, $h_{1}$ and $h_{2}$, is

$$
\left(h_{1} \mid h_{2}\right) \equiv 4 \int_{f_{\min }}^{f_{\mathrm{Ny}}} \frac{\tilde{h}_{1}(f) \tilde{h}_{2}^{*}(f)}{S_{n}(f)} \mathrm{d} f,
$$

where $S_{n}(f)$ is the one-sided power spectral density of the detector noise, which is assumed to be stationary and Gaussian with zero mean; $f_{\min }$ is the lower frequency cutoff for filtering, $f_{\mathrm{Ny}}$ is the Nyqyuist frequency corresponding to the waveform sampling rate, and $\tilde{h}(f)$ denotes the Fourier transform of $h(t)$. In this paper, we take $S_{n}(f)$ to be the zero-detuning high power noise curve for aLIGO, for both bank placement and overlap calculations [100] and set the lower-frequency cutoff $f_{\min }=15 \mathrm{~Hz}$. The peak GW frequency for the lowest binary masses that we consider, i.e., for $m_{1}+m_{2} \simeq 12 M_{\odot}$, is $\sim 2.1 \mathrm{kHz}$ during the ringdown phase. We sample the waveforms at $8192 \mathrm{~Hz}$, preserving the information content up to the Nyquist frequency $f_{\mathrm{Ny}}=4096 \mathrm{~Hz}$. A waveform, $h$, is normalized (made to be a unit vector) by $\hat{h}=h / \sqrt{h \mid h}$. In addition to being senstive to their intrinsic mass parameters, the inner product of two normalized waveforms is sensitive to phase and time shift differences between the two, $\phi_{c}$ and $t_{c}$. These two parameters $\left(\phi_{c}\right.$ and $\left.t_{c}\right)$ can be analytically maximized over to obtain the maximized overlap $\mathcal{O}$,

$$
\mathcal{O}\left(h_{1}, h_{2}\right)=\max _{\phi_{c}, t_{c}}\left(\left(\hat{h}_{1} \mid \hat{h}_{2}\left(\phi_{c}, t_{c}\right)\right)\right),
$$

which gives a measure of how "close" the two waveforms are in the waveform manifold, disregarding differences in overall amplitude. The mismatch $\mathcal{M}$ between the two waveforms is then

$$
\mathcal{M}\left(h_{1}, h_{2}\right)=1-\mathcal{O}\left(h_{1}, h_{2}\right) .
$$

Matched-filtering detection searches employ a discrete bank of modeled waveforms as filters. The optimal SNR is obtained when the detector strain $s(t) \equiv h^{\mathrm{tr}}(t)+n(t)$ is filtered with the true waveform $h^{\text {tr }}$ itself, i.e.,

$$
\rho_{\mathrm{opt}}=\max _{\phi_{c}, t_{c}}\left(\left(h^{\mathrm{tr}} \mid \hat{h}^{\mathrm{tr}}\left(\phi_{c}, t_{c}\right)\right)\right)=\left\|h^{\mathrm{tr}}\right\|,
$$

where $\left\|h^{\text {tr }}\right\| \equiv \sqrt{\left(h^{\text {tr }} \mid h^{\text {tr }}\right)}$ is the noise weighted norm of the waveform. The SNR $\rho$ recovered with a discrete bank of filter templates is

$$
\rho \simeq \mathcal{O}\left(h^{\mathrm{tr}}, h_{b}\right)\left\|h^{\mathrm{tr}}\right\|=\mathcal{O}\left(h^{\mathrm{tr}}, h_{b}\right) \rho_{\mathrm{opt}},
$$

where $h_{b}$ is the filter template in the bank (subscript $b$ ) that has the highest overlap with the signal $h^{\text {tr }}$. The furthest distance to which GW signals can be detected is proportional to the matched-filter SNR that the search algorithm finds the signal with. Note that $0 \leq \mathcal{O}\left(h^{\text {tr }}, h_{b}\right) \leq 1$, so the recovered SNR $\rho \leq \rho_{\text {opt }}$ [cf. Eq. (9)]. For a BBH population uniformly distributed in spatial volume, the detection rate would decrease as $\mathcal{O}\left(h^{\mathrm{tr}}, h_{b}\right)^{3}$. Searches that aim at restricting the loss in the detection rate strictly below $10 \%$ (or $15 \%$ ), would require a bank of template waveforms that have $\mathcal{O}$ above 0.965 (or 0.947) with any incoming signal $[101,102]$.

Any template bank has two sources for loss in SNR: (i) the discreteness of the bank grid in the physical parameter space of the BBHs and (ii) the disagreement between the actual GW signal $h^{\text {tr }}$ and the modeled template waveforms used as filters. We decoupled these to estimate the SNR loss. Signal waveforms are denoted as $h_{x}^{\text {tr }}$ in what follows, where the superscript tr indicates a true signal and the subscript $x$ indicates the mass parameters of the corresponding binary. Template waveforms are denoted as $h_{b}^{M}$, where $M$ denotes the waveform model and $b$ indicates that it is a member of the discrete bank. Figure 1 shows the signal $h_{x}^{\text {tr }}$ in its manifold and the bank of templates $h_{b}^{M}$ residing in the model waveform manifold, both being embedded in the same space of all possible waveforms. The point $h_{\perp}^{M}$ is the waveform which has the smallest mismatch in the entire (continuous) model manifold with $h_{x}^{\mathrm{tr}}$, i.e., $h_{\perp}^{M}: \mathcal{M}\left(h_{x}^{\mathrm{tr}}, h_{\perp}^{M}\right)=\min _{y} \mathcal{M}\left(h_{x}^{\mathrm{tr}}, h_{y}^{M}\right)$. The fraction of the optimal SNR recovered at different points $x$ in the binary mass space can be quantified by measuring the FF of the bank [73],

$$
\mathrm{FF}(x)=1-\min _{b} \mathcal{M}\left(h_{x}^{\mathrm{tr}}, h_{b}^{M}\right) .
$$

For two waveforms, $h_{1}$ and $h_{2}$, close to each other in the waveform manifold: $\left\|h_{1}\right\| \simeq\left\|h_{2}\right\|$, and mutually aligned in phase and time such that the overlap between them is maximized, 


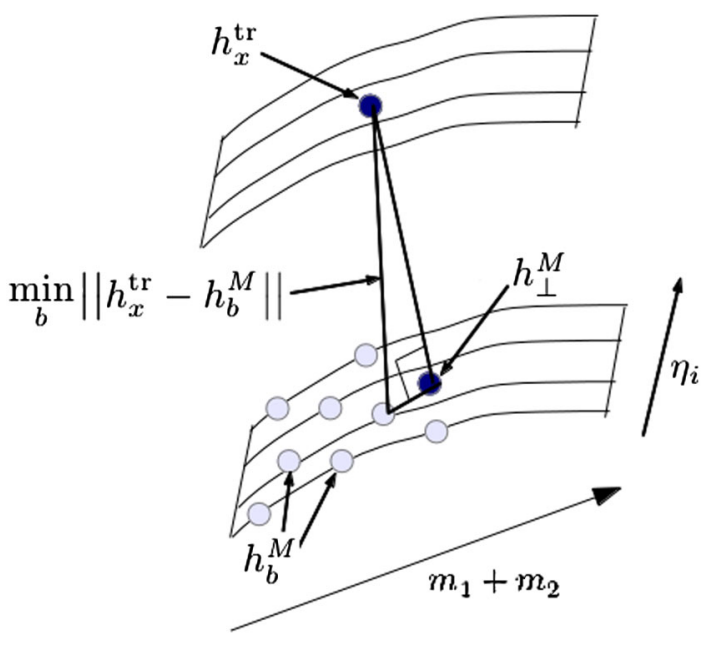

FIG. 1 (color online). We show the true (upper) and the hybrid (lower) waveform manifolds here, with the signal residing in the former, and a discrete bank of templates placed along lines of constant mass ratio in the latter. Both manifolds are embedded in the same space of all possible waveforms. The true signal waveform is denoted as $h_{x}^{\mathrm{tr}}$, while the templates in the bank are labelled $h_{b}^{M}$. The hybrid waveform that matches the signal $H_{x}^{\mathrm{tr}}$ best is shown as $h_{\perp}^{M}$. Also shown is the "distance" between the signal and the hybrid template that has the highest overlap with it. This figure is qualitatively similar to Fig. 3 of Ref. [101].

$$
\left\|h_{1}-h_{2}\right\|^{2} \simeq 2\left(h_{1} \mid h_{1}\right)\left(1-\frac{\left(h_{1} \mid h_{2}\right)}{\sqrt{\left(h_{1} \mid h_{1}\right)} \sqrt{\left(h_{1} \mid h_{1}\right)}}\right) .
$$

The mismatch can, hence, be written as [cf. Eq. (7)]

$$
\mathcal{M}\left(h_{1}, h_{2}\right)=\frac{1}{2\left\|h_{1}\right\|^{2}}\left\|h_{1}-h_{2}\right\|^{2}
$$

We note that this equation is an upper bound for Eq. (25) of Ref. [103]. From this relation, and treating the space embedding the true and model waveform manifolds as Euclidean at the scale of template separation, we can separate out the effects of bank coarseness and template inaccuracies as

$$
\begin{aligned}
\mathrm{FF}(x) & =1-\min _{b} \frac{1}{2\left\|h_{x}^{\mathrm{tr}}\right\|^{2}}\left\|h_{x}^{\mathrm{tr}}-h_{b}^{M}\right\|^{2}, \\
& =1-\Gamma_{\mathrm{Hyb}}(x)-\Gamma_{\mathrm{bank}}(x),
\end{aligned}
$$

where

$$
\Gamma_{\mathrm{Hyb}}(x) \equiv \frac{1}{2\left\|h_{x}^{\mathrm{tr}}\right\|^{2}}\left\|h_{x}^{\mathrm{tr}}-h_{\perp}^{M}\right\|^{2}=\mathcal{M}\left(h_{x}^{\mathrm{tr}}, h_{\perp}^{M}\right)
$$

is the SNR loss from model waveform errors out of the manifold of true signals and

$$
\Gamma_{\text {bank }}(x) \equiv \min _{b} \frac{1}{2\left\|h_{x}^{\mathrm{tr}}\right\|^{2}}\left\|h_{\perp}^{M}-h_{b}^{M}\right\|^{2}=\min _{b} \mathcal{M}\left(h_{\perp}^{M}, h_{b}^{M}\right)
$$

is the loss in the SNR from the distant spacing of templates in the bank. The decomposition in Eq. (13b) allows for the measurement of the two effects separately. NR-PN hybrids have the inspiral portion of the waveform, from PN theory, joined to the available late-inspiral and merger portions from NR (as described in Sec. II C). Toward the late inspiral, the PN waveforms accumulate phase errors, contaminating the hybrids [56,57]. For each hybrid, we constrain this effect using mismatches between hybrids constructed from the same NR simulation and different PN models, i.e.,

$$
\Gamma_{\mathrm{Hyb}}(x) \leq \mathcal{M}\left(h_{x}^{\mathrm{tr}}, h_{x}^{\mathrm{Hyb}}\right) \lesssim \max _{(i, j)} \mathcal{M}\left(h_{x}^{M_{i}}, h_{x}^{M_{j}}\right),
$$

where $M_{i}=$ TaylorT $[1,2,3,4]+$ NR. However, this is only possible for a few values of the mass ratio for which NR simulations are available. We assume $\Gamma_{\mathrm{Hyb}}$ to be a slowly and smoothly varying quantity over the componentmass space at the scale of template grid separation. At any arbitrary point $x$ in the mass space, we approximate $\Gamma_{\mathrm{Hyb}}$ with its value for the "closest" template, i.e.,

$$
\Gamma_{\mathrm{Hyb}}(x) \leq \max _{(i, j)} \mathcal{M}\left(h_{x}^{M_{i}}, h_{x}^{M_{j}}\right) \simeq \max _{(i, j)} \mathcal{M}\left(h_{b}^{M_{i}}, h_{b}^{M_{j}}\right),
$$

where $h_{b}^{M}$ is the hybrid template in the bank with the highest overlap with the signal at $x$.

The other contribution to SNR loss comes from the discrete placement of templates in the mass space. In Fig. 1, this is shown in the manifold of the template model. As NR waveforms (or hybrids) are available for a few values of the mass ratio, we measure this in the manifold of EOBNRv2 waveforms. The EOBNRv2 model reproduces most of the NR simulations that were consider here well [53], allowing for this approximation to hold. For the same reason, we expect $h_{x}^{\text {EOBNRv2 }}$ to be close to $h_{\perp}^{\mathrm{EOBNR} v 2}$, with an injective mapping between the two. This allows us to approximate [cf. Eq. (15)]

$$
\Gamma_{\text {bank }}(x) \simeq \min _{b} \mathcal{M}\left(h_{x}^{\mathrm{EOBNRv} 2}, h_{b}^{\mathrm{EOBNRv} 2}\right) .
$$

In Sec. IV, we construct template banks that use purely NR templates, which have negligible waveform errors. The SNR recovery from such banks is characterized with

$$
\mathrm{FF}(x)=1-\Gamma_{\text {bank }}(x),
$$

where the SNR loss from bank coarseness is obtained using Eq. (18). In Secs. V and VI, we construct template banks aimed at using NR-PN hybrid templates. Their SNR 
recovery is characterized using Eq. (13b), where the additional contribution from the hybrid waveform errors is obtained using Eq. (17).

\section{CONSTRUCTING A TEMPLATE BANK FOR NR WAVEFORMS}

In this section, we demonstrate the effectualness of a template bank viable for using NR waveforms as templates. The gravitational-wave phase of the dominant waveform multipole extracted from runs at different resolutions was found to converge within $\sim 0.3 \mathrm{rad}$ for $q=3,4,6$ and within $\sim 0.06 \mathrm{rad}$ for $q=1,2$ at merger (see Fig. 6 of Ref. [48], and Figs. 6 and 7 of Ref. [53] for a compilation). Most of this phase disagreement accumulates over a relatively short duration of $\sim 50 M-100 M$ before merger and is significantly lower over the preceding inspiral and plunge. As the matched-filter SNR accumulates secularly over the entire waveform, these numerical phase errors are negligible in terms of mismatches. We set $\Gamma_{\mathrm{Hyb}}=0$ while computing the fitting factors, so one is left with considering $\Gamma_{\text {bank }}$ to determine the fidelity of the bank [cf. Eq. (13b)].

With NR simulations as templates, the region that the bank can cover is restricted to binaries that have approximately the same number of waveform cycles within the sensitive frequency band of the detectors as the simulations themselves. We take their fiducial length to be $\sim 40 \mathrm{GW}$ cycles [104]. For BBHs with $3 M_{\odot} \leq m_{1}, m_{2} \leq 200 M_{\odot}$ and $m_{1}+m_{2} \leq 200 M_{\odot}$, we map out the region with $99 \%$ of the signal power within 40 cycles as the target region of the purely NR bank. For samples taken over the mass space, we determine the frequency interval $\left[f_{1}, f_{2}\right]$ for which

$$
\int_{f_{1}}^{f_{2}} \mathrm{~d} f \frac{|\tilde{h}(f)|^{2}}{S_{n}(|f|)}=0.99 \times \int_{f_{\min }}^{f_{\mathrm{Ny}}} \mathrm{d} f \frac{|\tilde{h}(f)|^{2}}{S_{n}(|f|)} .
$$

This is done by finding the peak of the integrand in Eq. (20) and integrating symmetrically outward from there, in time, till the interval $\left[f_{1}, f_{2}\right]$ is found. The number of waveform cycles in this interval is

$$
\mathrm{N}_{\mathrm{cyc}}=\frac{\Phi\left(t\left(f_{2}\right)\right)-\Phi\left(t\left(f_{1}\right)\right)}{2 \pi},
$$

where $\Phi(t)$ is the instantaneous phase of the waveform, $h_{+}(t)-\mathrm{i} h_{\times}(t)=A(t) e^{-\mathrm{i} \Phi(t)}$, unwrapped to be a monotonic function of time. We find that for a significant portion of the mass region, the signal power is contained within 40 waveform cycles. This is shown in Fig. 2, where the color at each point gives $\mathrm{N}_{\mathrm{cyc}}$ for that system, and the region with $\mathrm{N}_{\mathrm{cyc}}>40$ is excluded. Conservatively, this region is bounded by $\mathcal{M}_{c}=27 M_{\odot}$, as shown by the solid curve in the figure.

We place a bank over this region, using a stochastic method similar to Refs. [70-72]. The algorithm begins by

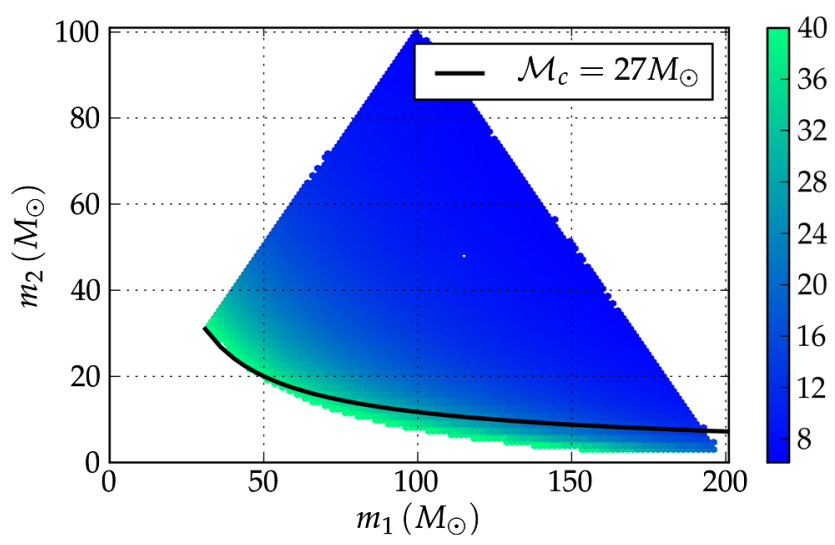

FIG. 2 (color online). The color at each point gives the number of waveform cycles, $\mathrm{N}_{\text {cyc }}$, for that particular binary, which contain $99 \%$ of the signal power in the aLIGO sensitivity band. The figure is truncated to exclude the region where $\mathrm{N}_{\mathrm{cyc}}>40$. The solid curve shows the lower bounding edge of the region with $\mathcal{M}_{c}=27 M_{\odot}$.

taking an empty bank, corresponding to step 0. At step $i$, a proposal point $(q, M)$ is picked by first choosing a value for $q$ from the restricted set $\mathcal{S}_{q}=\{1,2,3,4,6,8\}$. The total mass $M$ is subsequently sampled from the restricted interval corresponding to the predrawn $q$. The proposal is accepted if the waveform at this point has overlaps $\mathcal{O}<0.97$ with all the templates in the bank from step $i-1$. This gives the bank at step $i$. The process is repeated till the fraction of proposals being accepted falls below $\sim 10^{-4}$, and $\gtrsim 99 \%$ of the parameter space is covered effectually. To complete the coverage, 100,000 points are sampled over the region of mass space depicted in Fig. 2, and the FF of the bank is computed at each point. With the islands of undercoverage isolated, the points sampled in these regions are added to the bank, pushing their mass ratios to the two neighboring mass ratios in $\mathcal{S}_{q}$ along lines of constant chirp mass. This helps accelerate the convergence of the bank, albeit at the cost of overpopulating it, as the algorithm for computing the FF for the sampled points is parallelizable.

We asses the effectualness of the bank, as discussed in Sec. III, using Eq. (19). We draw a population of 100,000 $\mathrm{BBH}$ signals, uniformly from the binary mass space, and filter them through the bank. Figure 3 shows the FF, or the fraction of the optimal SNR recovered by the bank. The region shown is restricted to binaries with $N_{\text {cyc }} \leq 40$. The black dots in the figure show the position of templates in the bank. The bank recovers $\geq 97 \%$ of the optimal SNR over the entire region of interest for $q \leq 10$. We propose an additional simulation for $q=9.2$, to increase the coverage to higher mass ratios. Substituting this for $q=8$ in the set of allowed mass ratios $\mathcal{S}_{q}$, we place another bank as before, with $\mathcal{S}_{q}=\{1,2,3,4,6,9.2\}$. The SNR loss from this bank is shown in Fig. 4. This bank recovers $\geq 97 \%$ of the SNR for systems with $q \leq 11$ and $\mathrm{N}_{\mathrm{cyc}} \leq 40$. The choice of the 
PRAYUSH KUMAR

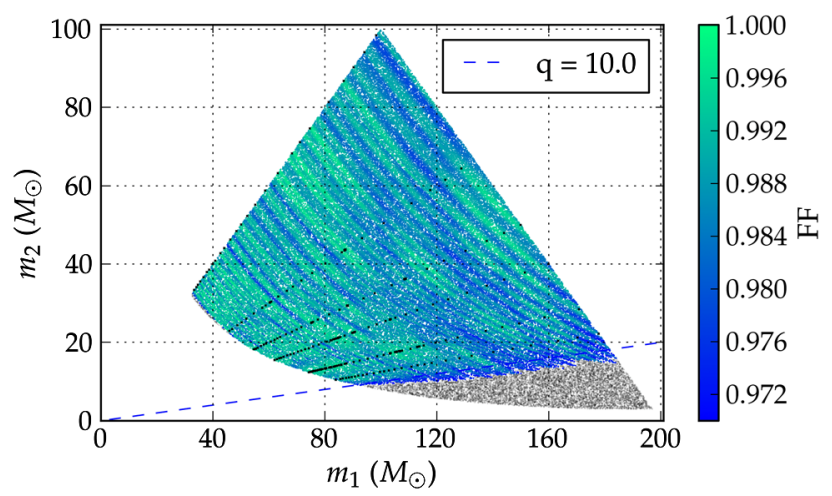

FIG. 3 (color online). The color at each point in the figure gives the value of $\mathrm{FF} \simeq 1-\Gamma_{\text {bank }}$ of the bank for that binary, for the NR bank restricted to $\mathcal{S}_{q}=\{1,2,3,4,6,8\}$. This is the same as the fraction of the optimal SNR, for the binary, that the template bank recovers. The black dots show the location of the templates in the bank. We note that they all lie along straight lines of constant $q$ passing through the origin. The region shaded light grey (toward the bottom of the figure) is where the FF drops sharply below $97 \%$.

additional simulation at $q=9.2$ was made by choosing a value close to the highest possible value of $q$ that does not lead to undercoverage in the region between $q=6$ and that value. The exact highest allowed value was not chosen to reduce the sensitivity of the coverage of the bank to fluctuations in detector sensitivity.

We conclude that with only six NR waveforms for nonspinning BBHs, which are 20 orbits (or $40 \mathrm{GW}$ cycles) in length, a template bank can be constructed that is effectual for detecting binaries with chirp mass above $27 M_{\odot}$ and $1 \leq q \leq 10$. With an additional simulation for

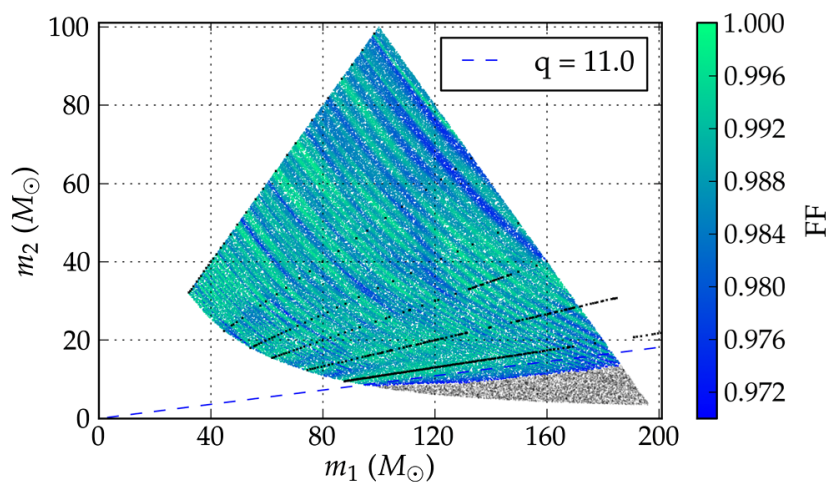

FIG. 4 (color online). This figure is similar to Fig. 3. The color at each point gives the value of $\mathrm{FF} \simeq 1-\Gamma_{\text {bank }}$ of the bank for that binary, for the NR bank restricted to $\mathcal{S}_{q}=\{1,2,3,4,6,9.2\}$. The black dots show the location of the templates in the bank. The region shaded light grey (toward the bottom of the figure) is where the FF drops below 97\%. We note that with an additional NR waveform for mass ratio $q=9.2$, the coverage of the bank is extended to include binaries with $10 \leq q \leq 11$. $q=9.2$, this bank can be extended to higher mass ratios, i.e., to $1 \leq q \leq 11$.

\section{CONSTRUCTING A TEMPLATE BANK FOR NR-PN HYBRIDS}

The template bank constructed in Sec. IV is effectual for GW detection searches focused at relatively massive binaries with $\mathcal{M}_{c} \gtrsim 27 M_{\odot}$. As the NR waveforms are restricted to a small number of orbits, it is useful to consider NR-PN hybrids to bring the lower mass limit down on the template bank. PN waveforms can be generated for an arbitrarily large number of inspiral orbits, reasonably accurately and relatively cheaply. Thus, a hybrid waveform comprised of a long PN early inspiral and an NR late inspiral, merger, and ringdown could also be arbitrarily long. There are, however, uncertainties in the PN waveforms, due to the unknown higher-order terms. During the late-inspiral and merger phases, these terms become more important, and the PN description becomes less accurate. In addition, when more of the late inspiral is in the detector's sensitivity frequency range, hybrid waveform mismatches due to the PN errors become increasingly large and reduce the recovered SNR. Thus, when hybridizing PN and NR waveforms, there must be enough NR orbits that the PN error is sufficiently low for the considered detector noise curve. In this section, we construct an NR + PN hybrid template bank, for currently available NR waveforms, and determine the lowest value of binary masses to which it covers.

The hybrids we use are constructed by joining the PN and NR portions, as described in Sec. II C. The number of orbits before merger at which they are joined depends on the length of the available NR waveforms. We estimate the PN waveform errors using hybridization mismatches $\Gamma_{\mathrm{Hyb}}$, as discussed in Sec. III. Figure 5 shows the same for all the hybrids, as a function of total mass. In terms of orbital frequency, these are matched at $M \omega_{m}=0.025$ for $q=1$, $M \omega_{m}=0.038$ for $q=2$, and $M \omega_{m}=0.042$ for $q=3,4$, 6,8 . In terms of the number of orbits before merger, this is 26.9 orbits for $q=1,13.6$ orbits for $q=2,12.6$ orbits for $q=3,14.3$ orbits for $q=4,17.8$ orbits for $q=6$, and 21.4 orbits for $q=8$. The dotted line indicates a mismatch of $1.5 \%$, a comparatively tight bound that leaves flexibility to accommodate errors due to template bank discreteness. The black circles show the hybrid mismatches at the lower mass bound of the NR-only template bank in Table I, which are negligible. The inset shows this minimum mass as a function of mass ratio, as well as the minimum attainable mass if we accept a hybrid error of $1.5 \%$. At lower masses, the mismatches increase sharply with more of the PN part moving into the Advanced LIGO sensitivity band. This is due to the nature of the frequency dependence of the detector sensitivity. The detectors will be most sensitive in a comparatively narrow frequency band. As the hybridization frequency sweeps through this band, the hybrid errors rise 


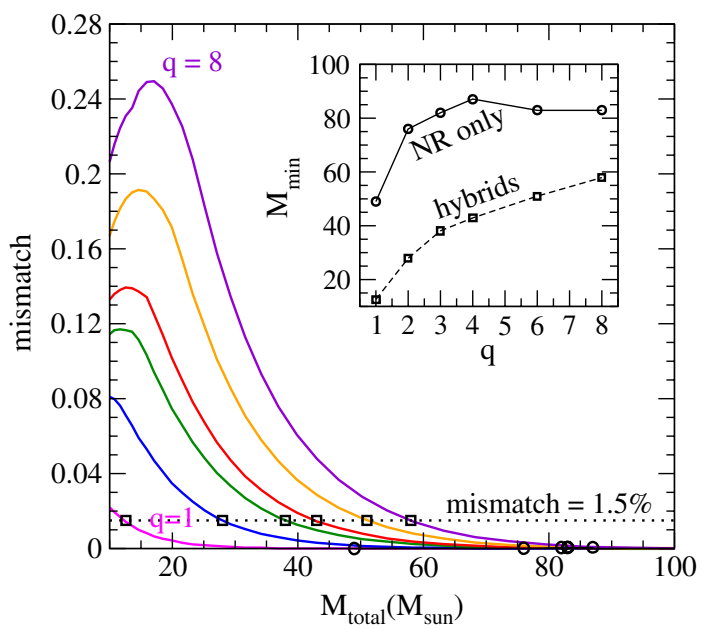

FIG. 5 (color online). Bounds on mismatches of NR-PN hybrid waveforms, for the currently existing NR simulations. The PN error is for hybrids matched at $M \omega_{m}=0.025$ for $q=1, M \omega_{m}=0.038$ for $q=2$, and $M \omega_{m}=0.042$ for $q=3,4,6,8$. The black circles indicate the lower bound of the template bank in Table I. The black squares show the lower bound with a hybrid error of $1.5 \%$. The inset shows these lower bounds as a function of mass ratio.

sharply. They fall again at the lowest masses, for which mostly the PN portion stays within the sensitive band.

We now consider template banks viable for hybrids constructed from currently available NR waveforms at mass ratios $q=1,2,3,4,6,8$. The lower mass limit, in this case, is extended down to masses where the hybridization error exceeds $3 \%$. We demonstrate two independent methods of laying down the bank grid. First, we use the stochastic placement method that proceeds as described in Sec. IV. The templates are sampled over the total mass and mass ratio $(M, q)$ coordinates, sampling $q$ from the restricted set. The total mass $M$ is sampled from the continuous interval between the lower mass limit, which is different for each $q$, and the upper limit of $200 M_{\odot}$. To assess the SNR loss from the sparse placement of the templates, we simulate a population of $100,000 \mathrm{BBH}$ signal waveforms, with masses sampled with $3 M_{\odot} \leq m_{1,2} \leq$ $200 M_{\odot}$ and $M \leq 200 M_{\odot}$, and filter them through the bank. This portion of the SNR loss needs to be measured with both signals and templates in the same waveform manifold. We use the EOBNRv2 approximant [53] to model both, as it has been calibrated to most of the NR waveforms we consider here, and it allows us to model waveforms for arbitrary systems. The left panel of Fig. 6 shows the fraction of the optimal SNR that the bank recovers, accounting for its discreteness alone. We observe that, with just six mass ratios, the bank can be extended to much lower masses before it is limited by the restricted sampling of mass ratios for the templates. For binaries with both black holes more massive than $\sim 12 M_{\odot}$, the spacing between mass ratios was found to be sufficiently dense. The total SNR loss, after subtracting out the hybrid mismatches from Fig. 5, are shown in the right panel of Fig. 6. At the lowest masses, the coverage shrinks between the lines of constant $q$ over which the templates are placed, due to the hybrid errors increasing sharply. We conclude that this bank is viable for using hybrid templates in $\mathrm{GW}$ searches for BBHs with $m_{1,2} \geq 12 M_{\odot}, 1 \leq q \leq 10$, and $M \leq 200 M_{\odot}$. Over this region, the bank will recover more than $96.5 \%$ of the optimal SNR. This is a significant increase over the coverage allowed for with the purely NR bank, the region of coverage of which is shown in the right panel of Fig. 7, bounded at lowest masses by the magenta (solid) curve.

Second, we demonstrate a nonstochastic algorithm of bank placement, with comparable results. We first construct
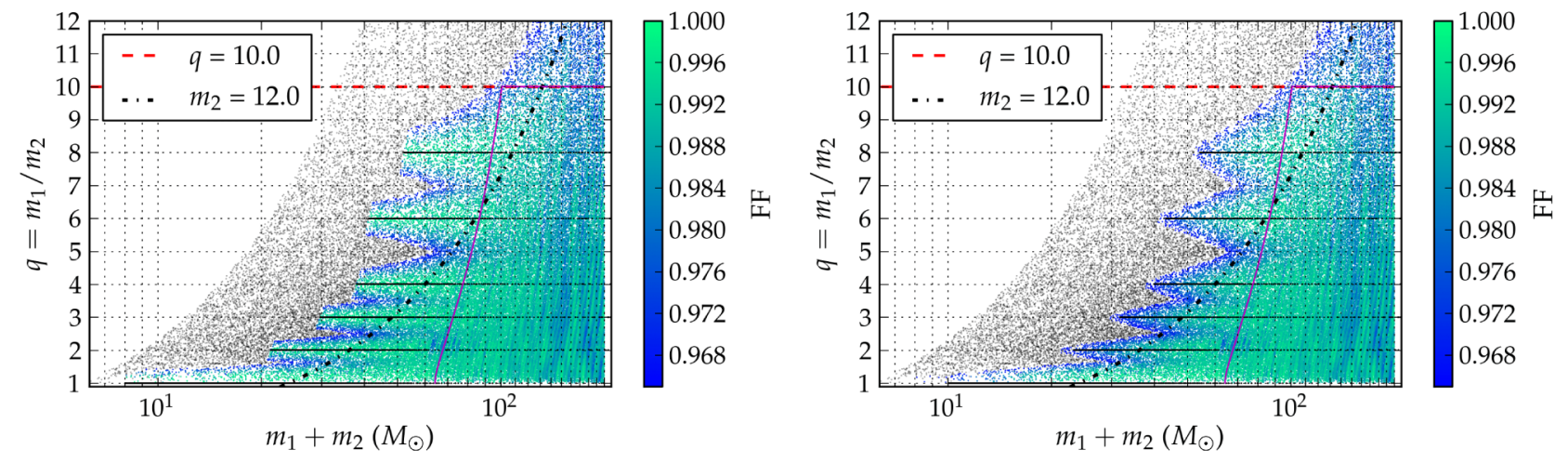

FIG. 6 (color online). These figures show fitting factors FF obtained when using a discrete mass-ratio template bank for $q=1,2,3,4$, 6 , 8. For each mass ratio, the templates are extended down to a total mass where the NR-PN hybridization mismatch becomes $3 \%$. The bank is placed using the stochastic algorithm, similar to Refs. [70-72]. The black dots show the location of the templates. The fitting factor on the left plot does not take into account the hybridization error and therefore shows the effect of the sparse placement of the templates alone. The right plot accounts for the hybridization error and gives the actual fraction of the optimal SNR that would be recovered with this bank of NR-PN hybrid templates. The region bounded by the magenta (solid) line in both plots indicates the lower end of the coverage of the bank of unhybridized NR waveforms. Lastly, the shaded grey dots show the points where the fitting factor was below $96.5 \%$. 

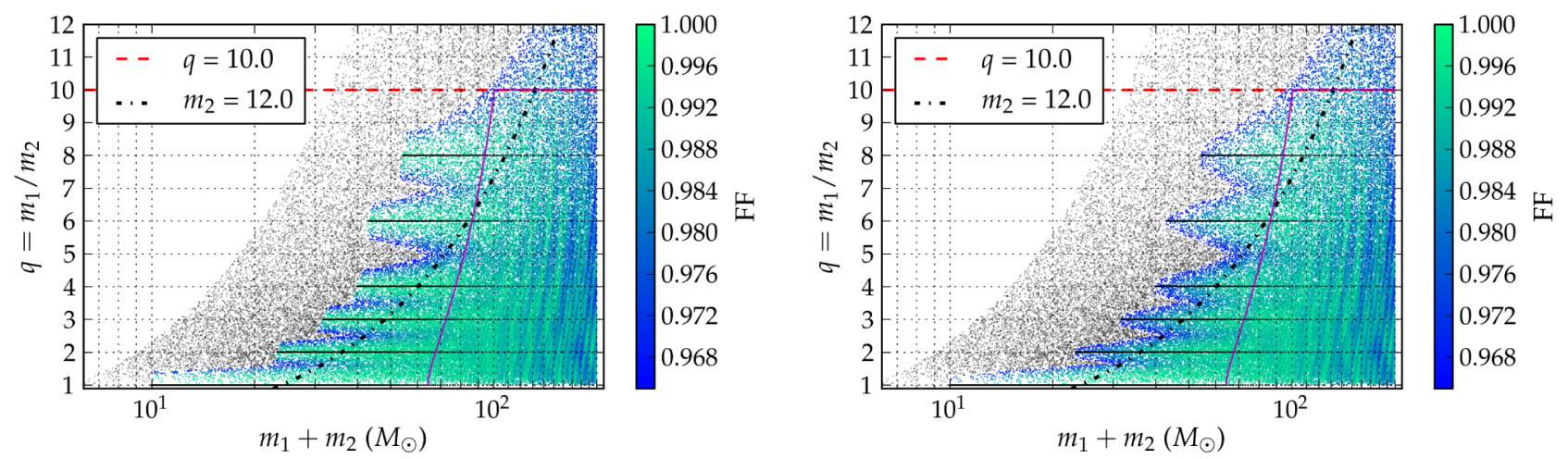

FIG. 7 (color online). These figures are similar to Fig. 6. The figures show FFs obtained when using a discrete mass-ratio template bank for $q=1,2,3,4,6,8$. For each mass ratio, the templates are extended down to a total mass where the NR-PN hybridization mismatch becomes $3 \%$. Templates are placed independently for each mass ratio and span the full range of total masses. For each mass ratio, neighboring templates are required to have an overlap of $97 \%$. The union of the six single- $q$ one-dimensional banks is taken as the final bank. The black dots show the location of the templates. The fitting factor on the left plot does not take into account the hybridization error and therefore shows the effect of the sparse placement of the templates alone. The right plot accounts for the hybridization error and gives the actual fraction of the optimal SNR that would be recovered with this bank of NR-PN hybrid templates. The region bounded by the magenta (solid) line in both plots indicates the lower end of the coverage of the bank of unhybridized NR waveforms. Lastly, the shaded grey dots show the points where the fitting factor was below $96.5 \%$.

six independent bank grids, each restricted to one of the mass ratios $q=1,2,3,4,6,8$ and spanning the full range of total masses. The template with the lowest total mass is chosen by requiring the hybrid mismatch to be $3 \%$ at that point. The spacing between neighboring templates is given by requiring that the overlap between them be $97 \%$. We take the union of these banks as the final two-dimensional bank. As before, we measure the SNR loss due to discreteness of the bank and the waveform errors in the templates separately. To estimate the former, we simulate a population of $100,000 \mathrm{BBH}$ systems and filter them through the bank. The signals and the templates are both modeled with the EOBNRv2 model. The left panel of Fig. 7 reveals the fraction of SNR recovered over the mass space, accounting for the sparsity of the bank alone, i.e., $1-\Gamma_{\text {bank }}$. At lower masses, we again start to see gaps between the lines of constant mass ratio which become significant at $m_{1,2} \leq 12 M_{\odot}$. The right panel of Fig. 7 shows the final fraction of the optimal SNR recovered, i.e., the FF as defined in Eq. (13b). As before, these are computed by
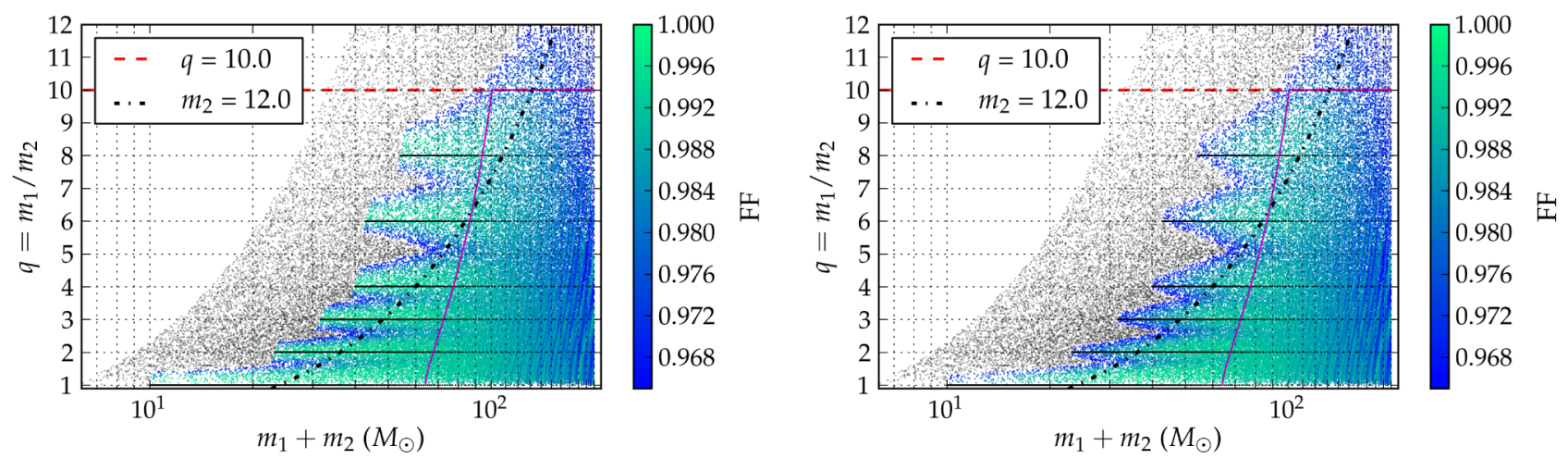

FIG. 8 (color online). This figure is similar to Fig. 7. The figures show FFs obtained when using a discrete mass-ratio template bank for $q=1,2,3,4,6,8$. Templates are placed independently for each mass ratio and span the range of total masses, down to the region where the hybrid errors become $3 \%$. For each mass ratio, neighboring templates are required to have an overlap of $97 \%$. The union of the six single- $q$ one-dimensional banks is taken as the final bank. The black dots show the location of the templates. The GW signals are modeled using the EOBNRv2 approximant [53], while TaylorT4 + NR hybrids are used as templates. The fitting factor on the left plot shows the combined effect of the sparse placement of the templates and the (relatively small) disagreement between the hybrid and EOBNRv2 waveforms. The right plot explicitly accounts for the hybridization error and gives the (conservative) actual fraction of the optimal SNR that would be recovered with this bank of NR-PN hybrid templates. The region bounded by the magenta (solid) line in both plots indicates the lower end of the coverage of the bank of unhybridized NR waveforms. Lastly, the shaded grey dots show the points where the fitting factor was below $96.5 \%$. 
subtracting out the hybrid mismatches $\Gamma_{\mathrm{Hyb}}$ in addition to the discrete mismatches, as described in Sec. III.

The efficacy of both methods of template bank construction can be compared from Figs. 6 and 7. We observe that the final banks from either of the algorithms have very similar SNR recovery and are both effectual over the range of masses we consider here. Both were also found to give a very similar number of templates. The uniform-in-overlap method yields a grid with 2,325 templates. The stochastic bank, on the other hand, was placed with a requirement of 98\% minimal mismatch and had 2,457 templates. This, however, includes templates with $m_{1,2}<12 M_{\odot}$. Restricted to provide coverage over the region with $m_{1,2} \geq 12 M_{\odot}$, $1 \leq q \leq 10$, and $M \leq 200 M_{\odot}$, the two methods yield banks with 627 and 667 templates, respectively. The size of these banks is comparable to one constructed using the second-order post-Newtonian TaylorF2 hexagonal template placement method [32,34-36], which yields a grid of 522 and 736 templates for a minimal match of $97 \%$ and $98 \%$, respectively.

Finally, we test the robustness of these results using TaylorT4 + NR hybrids as templates. As before, we simulate a population of $100,000 \mathrm{BBH}$ signal waveforms. As we do not have hybrids for arbitrary binary masses, we model the signals as EOBNRv2 waveforms. This population is filtered against a bank of hybrid templates. The SNR recovered is shown in the left panel of Fig. 8. Comparing with the left panels of Figs. 6 and 7, we find that the EOBNRv2 manifold is a reasonable approximation for the hybrid manifold and that, at lower masses, there is a small systematic bias in the hybrids toward EOBNRv2 signals with slightly higher mass ratios. The right panel of Fig. 8 shows the fraction of optimal SNR recovered after subtracting out the hybrid mismatches from the left panel. The similarity of the FF distribution between the right panels of Fig. 8 and Figs. 6 and 7 is remarkable. This gives us confidence that the EOBNRv2 model is a good approximation for testing NR/hybrid template banks, as we do in this paper, and that a template bank of NR + PN hybrids is indeed effectual for binaries with $m_{1,2} \geq 12 M_{\odot}$, $M \leq 200 M_{\odot}$, and $q \leq 10$.

\section{COMPLETE NR-PN HYBRID BANK FOR NONSPINNING BBH}

The last sections outlined properties of template banks of NR waveforms (and their hybrids) which are available today. We also investigate the parameter and length requirements for future NR simulations, which would let us construct detection template banks all the way to $M=m_{1}+m_{2}=$ $12 M_{\odot}$. This lower limit was chosen following Refs. [66,67], which showed that the region with $M \lesssim 12 M_{\odot}$ can be
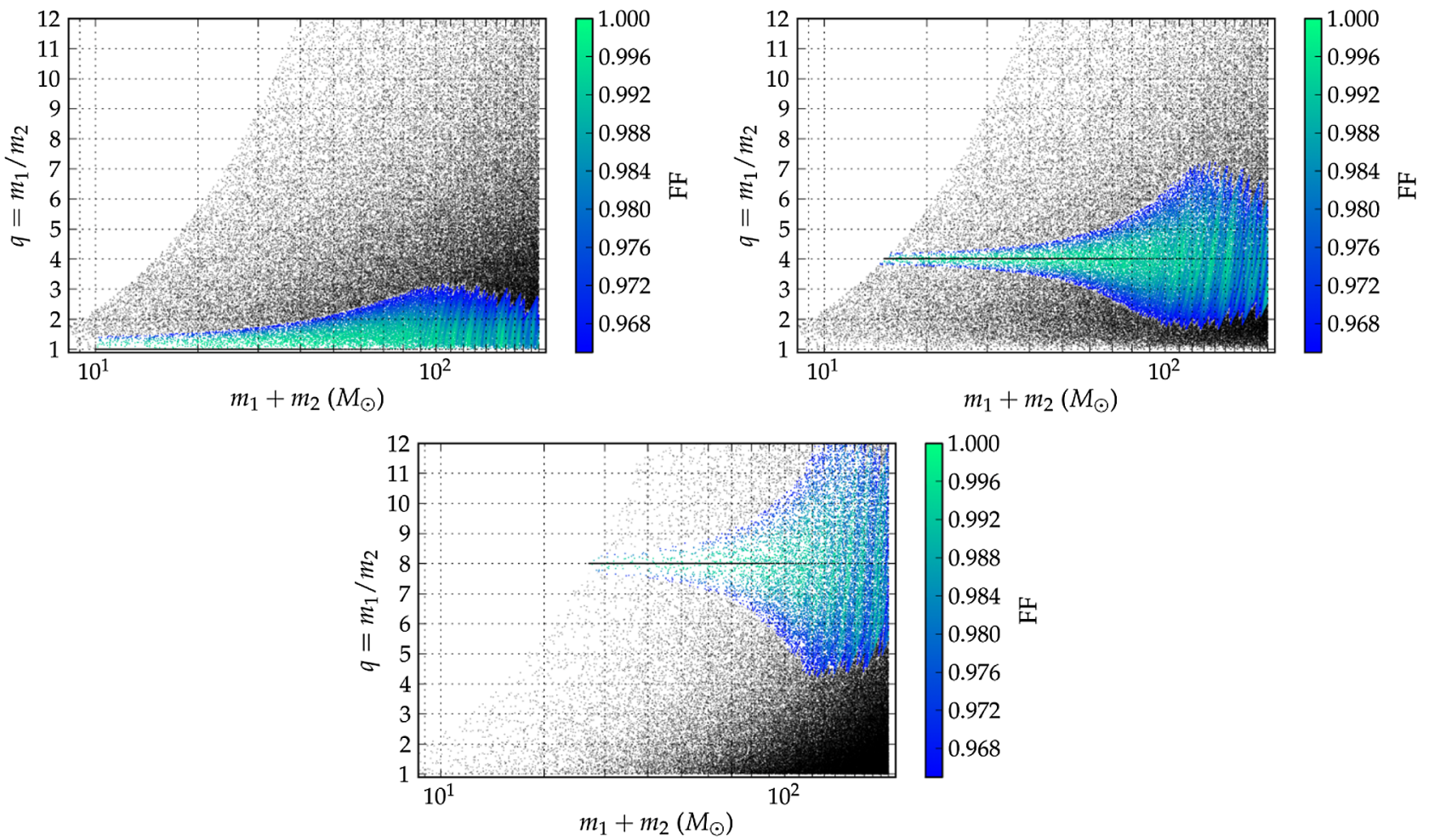

FIG. 9 (color online). These figures show the coverage of template banks restricted to single mass ratios, i.e., (from left to right) $q=1$, 4,8 . We note that at higher total masses, the templates are correlated to simulated signals for considerably different mass ratios than at lower total masses. This agrees with what we expect as with decreasing total mass: the number of cycles in the sensitive frequency band of Advanced LIGO increases. 
TABLE II. List of mass ratios, a template bank restricted to which will be effectual over the region of the nonspinning $\mathrm{BBH}$ mass space where $m_{1}+m_{2} \gtrsim 12 M_{\odot}$ and $1 \leq q \leq 10$. The fraction of the optimal SNR recovered by such a bank, accounting for discreteness losses, remains above 98\%. This is shown in Fig. 10.

\begin{tabular}{c}
\hline \hline$q\left(\equiv m_{1} / m_{2}\right)$ \\
\hline $1,1.5,1.75$, \\
$2,2.25,2.5,2.75$, \\
$3,3.25,3.5,3.8$, \\
$4.05,4.35,4.65,4.95$, \\
$5.25,5.55,5.85$, \\
$6.2,6.55$, \\
$7,7.5$, \\
$8,8.5$, \\
$9,9.6$ \\
\hline \hline
\end{tabular}

covered with banks of post-Newtonian inspiral-only waveforms.

Constructing such a bank is a two-step process. First, we pick mass ratios that allow construction of such a bank given long enough waveforms for these mass ratios. Second, one needs to determine the necessary length of the NR portion of the waveforms, such that the PN-hybridization error is sufficiently low for all masses of interest.

To motivate the first step, Fig. 9 shows the coverage of banks that sample from a single mass ratio each (from left to right: $q=1,4,8$ ). We see that the resolution in $q$ required at lower values of $M$ increases sharply below $M \sim 60 M_{\odot}$. This follows from the increase in the number of waveform cycles in aLIGO frequency band as the total mass decreases, which, in turn, increases the discriminatory resolution of the matched filter along the $q$ axis. To determine the least set of mass ratios which would sample the $q$ axis sufficiently densely at lower masses, we iteratively add mass ratios to the allowed set and test banks restricted to sample from it. We find that, constrained to the set $\mathcal{S}_{q}$ given in Table II, a template bank can be constructed that has a minimal match of $98 \%$ at the lowest masses. The left panel of Fig. 10 shows the loss in SNR due to bank grid coarseness, i.e., $1-\Gamma_{\text {bank }}$. This loss remains below $2 \%$ for mass ratios $1 \leq q \leq 10$, even at $M=12 M_{\odot}$. This leaves a margin of $1.5 \%$ for the hybrid mismatches that would incur due to the hybridization of the NR merger waveforms with long PN inspirals. The right panel of Fig. 10 shows the same data in the $m_{1}-m_{2}$ plane. In this figure, the region covered by the NR-only bank is above the blue (solid) curve, while that covered by a bank of the currently available NR-PN hybrids is above the line of $m_{2}=12 M_{\odot}$ (with $m_{2} \leq m_{1}$ ). The region from Refs. $[66,67]$ that can be covered by PN templates is in the bottom left corner, bounded by the magenta (solid) line. Our bank restricted to the set of 26 mass ratios, as above, provides additional coverage for binaries with $M \geq 12 M_{\odot}$, $m_{2} \leq 12 M_{\odot}$, and $1 \leq q \leq 10$. Thus, between purely PN and NR/NR-PN hybrid templates, we can construct effectual searches for nonspinning BBHs with $q \leq 10$.

Having the set of required mass ratios $\mathcal{S}_{q}$ determined, we need to decide on the length requirements for the NR simulations, in order to control the PN hybridization error. For a series of matching frequencies, we construct NR-PN hybrids with Taylor $\{\mathrm{T} 1, \mathrm{~T} 2, \mathrm{~T} 3, \mathrm{~T} 4\}$ inspirals and compute their pairwise mismatch as a function of total mass. The maximum of these mismatches serves a conservative bound on the PN-hybridization error for that hybrid [cf. Eq. (17)]. Figure 11 shows the results of this calculation. Each panel of Fig. 11 focuses on one mass
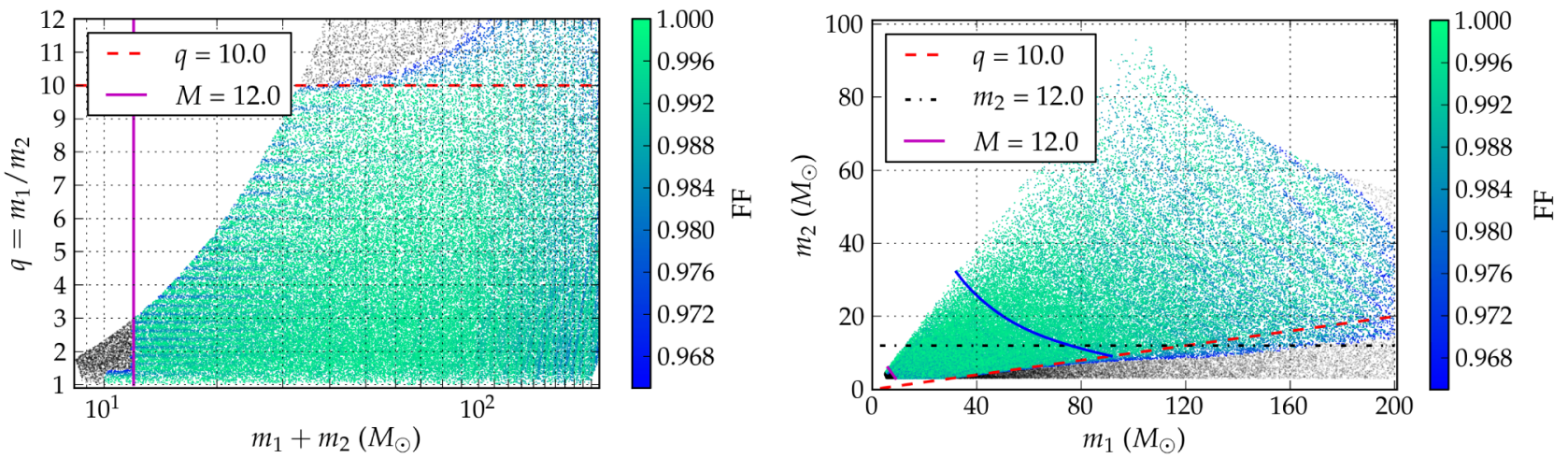

FIG. 10 (color online). This figure shows fitting factors for a hybrid template bank which samples from the 26 mass ratios $q=1,1.5,1.75,2, \ldots, 9.6$ and allows coverage to masses down to $m_{1}+m_{2}=12 M_{\odot}$ and $1 \leq q \leq 10$, with a minimal match of $98 \%$ at the lowest masses. The left and right panels show the same on the $M-q$ and $m_{1}-m_{2}$ axes, respectively. The magenta lines, in both panels, show the upper bound in total mass, below which frequency-domain PN waveforms can be used to construct template banks for aLIGO searches $[66,67]$. The dashed-dotted line in the right panel shows the lower mass limit on the smaller component object, to which a bank of currently available NR-PN hybrids can cover, i.e., $\min \left(m_{1}, m_{2}\right)=12 M_{\odot}$ (see Sec. V). The blue (solid) curve in the right panel gives the lower mass limit to which a bank of currently available NR waveforms can cover (see Sec. IV). Thus, between the simulations listed in Table II and frequency domain PN waveforms, we can search for the entire range of BBH masses. 

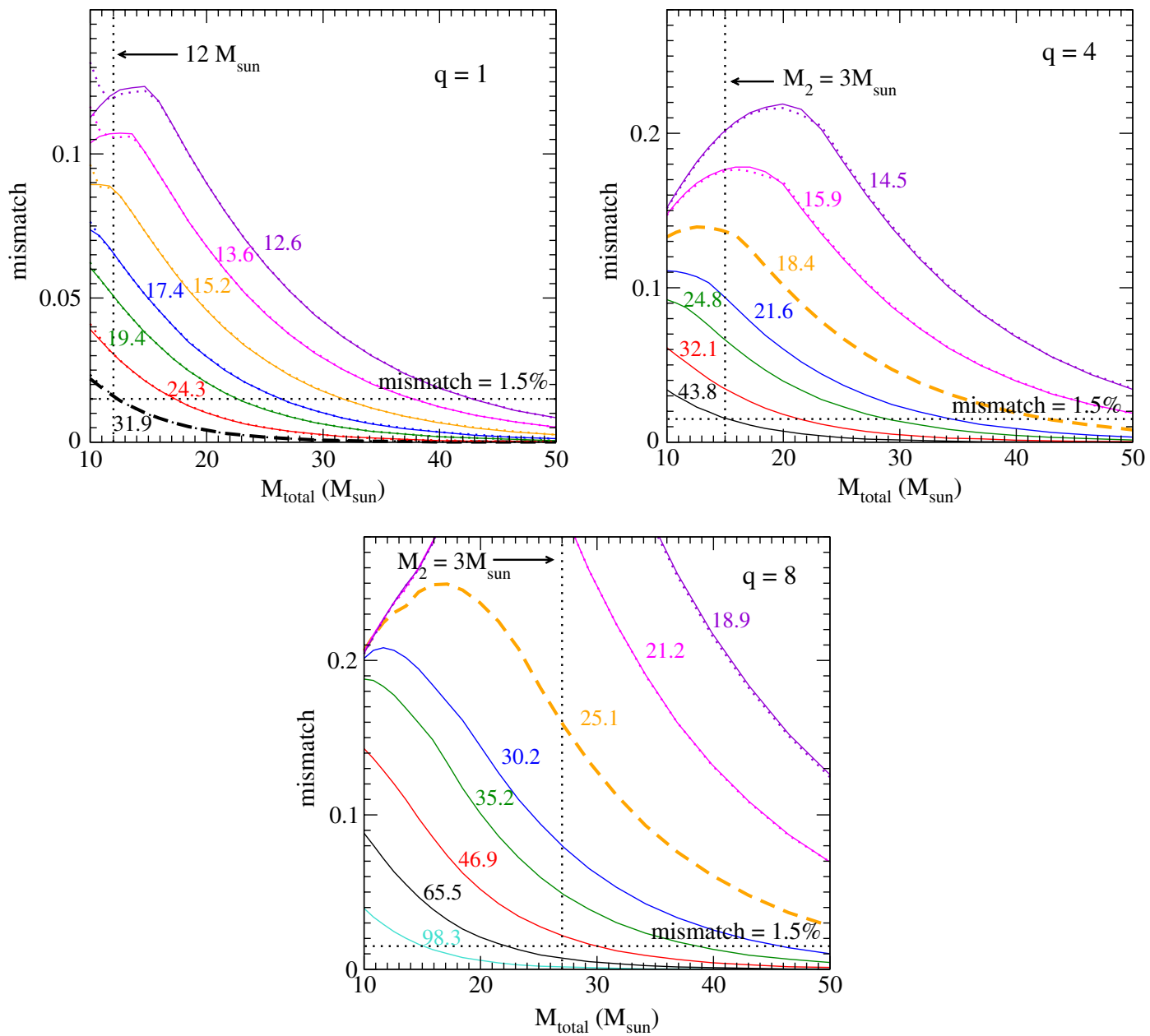

FIG. 11 (color online). The curves show the maximum mismatch between hybrids constructed with different PN approximants, at different matching frequencies $\left(M \omega_{m}\right)$, as a function of the binary total mass. The dotted lines indicate a mismatch of $1.5 \%$ and a lower total mass limit, $12 M_{\odot}$ for $q=1$, and $M_{2}=3 M_{\odot}$ for $q=4,8$. The thick dashed lines indicate the currently possible matching frequency for hybrids based on the length of NR waveforms. The numbers next to each line indicate the number of orbits before merger where the PN and NR (or EOB) waveforms were stitched together.

ratio. Within each panel, each line represents one matching frequency, with lines moving down toward earlier hybridization with smaller mismatches. Because the hybridization frequency is not particularly intuitive, the lines are labeled by the number of orbits of the NR portion of the hybrid waveform. For a small number of orbits, this calculation is indeed done with NR waveforms, whereas for a large number of orbits, we substitute EOBNRv2 waveforms. The dashed lines represent the earliest one can match a NR + PN hybrid given the currently available NR waveforms and are the same as the $q=1,4$, and 8 lines in Fig. 5. The solid curves show the results using EOB hybrids, while the dotted curves (just barely visible) show the results with NR hybrids. They are virtually identical, which is a confirmation that EOB hybrids can act as a good proxy for NR hybrids in this case. The horizontal dotted line indicates a mismatch of $1.5 \%$, while the vertical dotted line shows a lower mass limit for each mass ratio: $12 M_{\odot}$ for $q=1$, which is the point at which one can construct a template bank with only PN inspirals, $15 M_{\odot}$ for $q=4$ and $27 M_{\odot}$ for $q=8$, which are the lower mass limits if both component masses are $\geq 3 M_{\odot}$.

Figure 12 presents the information obtained in the previous paragraph in a different way. Given NR-PN hybrids with $N$ orbits of NR, the shaded areas in the left panel of Fig. 12 indicate the region of parameter space for which such hybrids have hybridization errors smaller than $1.5 \%$. As before, we see that for high masses, comparatively few NR orbits are sufficient (e.g., the purple $N=15$ region), whereas lower total masses require increasingly more NR orbits. The dashed lines indicate the region of parameter space with hybrid error below 3\%. The black dotted line designates the point where one component mass is greater than $3 M_{\odot}$, which is a reasonable lower mass limit for a physical black hole. The right panel shows this same analysis instead with the initial GW frequency indicated by 

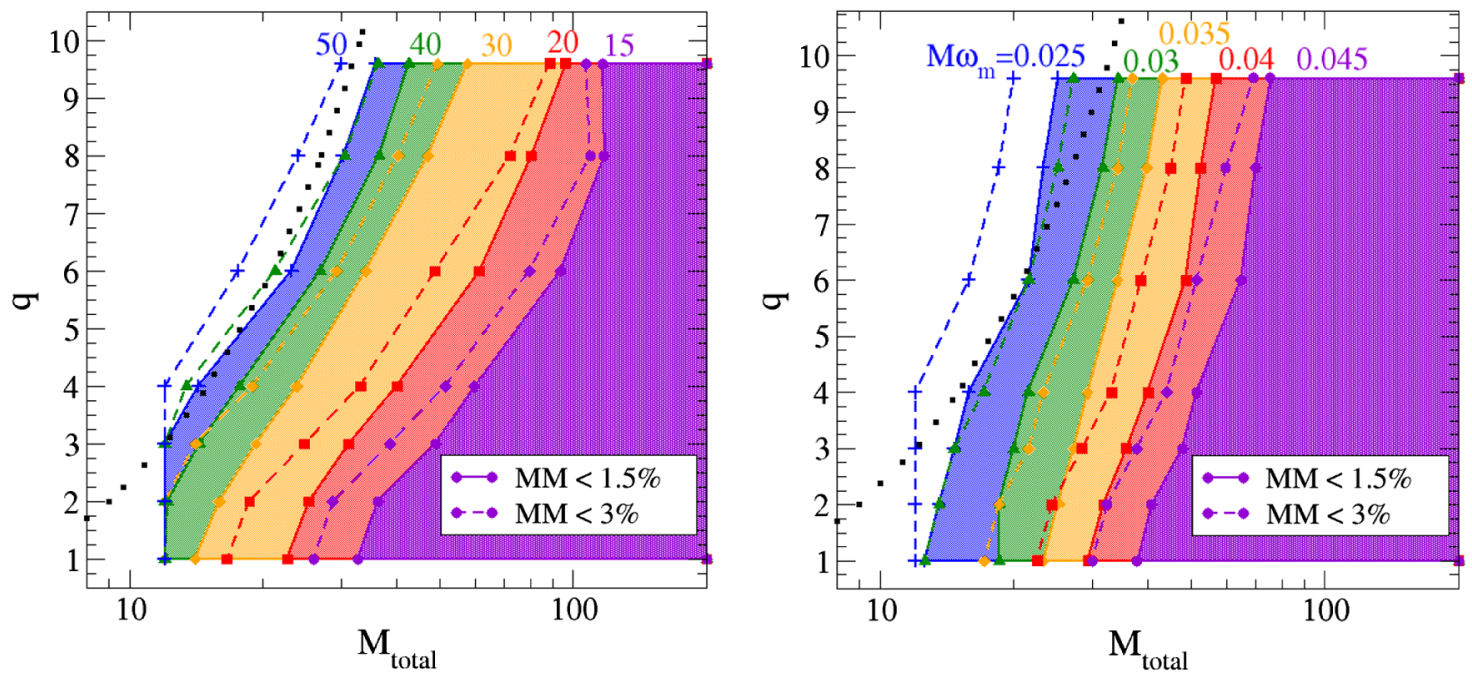

FIG. 12 (color online). This plot shows the lower mass limit of a template bank constructed with hybrid waveforms in terms of the number of NR orbits (left panel) and initial gravitational wave frequency (right panel) needed to have a PN error below 1.5\% (solid curves) or $3 \%$ (dashed curves). The dotted line indicates the lower total mass limit when one component mass is $3 M_{\odot}$.

the solid and dashed lines. Thus, for the region of parameter space we are interested in, no more than $\sim 50 \mathrm{NR}$ orbits or an initial GW frequency of $M \omega=0.025$ would be necessary to construct a detection bank with hybrid mismatches below $1.5 \%$.

\section{CONCLUSIONS}

The upgrades currently being installed to increase the sensitivity of the ground-based interferometric gravitational-wave detectors LIGO and Virgo [1,2] are scheduled to complete within the next two years. The second generation detectors will have a factor of 10 better sensitivity across the sensitive frequency band, with the lower frequency limit being pushed from $40 \mathrm{~Hz}$ down to $\sim 10 \mathrm{~Hz}$. They will be able to detect GWs from stellar-mass BBHs up to distances of a few $\mathrm{Gpc}$, with the expected frequency of detection between 0.4-1000 $\mathrm{yr}^{-1}$ [5].

Gravitational-wave detection searches for BBHs operate by matched filtering the detector data against a bank of modeled waveform templates [31-36,105]. Early LIGOVirgo searches employed PN waveform template banks that spanned only the inspiral phase of the coalescence [16-20]. Recent work has shown that a similar bank of PN templates would be effectual for the advanced detectors, to detect nonspinning BBHs with $m_{1}+m_{2} \lesssim 12 M_{\odot} \quad[66,67]$. Searches from the observation period between 2005-2007 and 2009-2010 employed templates that also included the late-inspiral, merger, and ringdown phases of binary coalescence [21,22].

Recent advancements in numerical relativity have led to high-accuracy simulations of the late inspiral and mergers of BBHs. The multidomain SpEC code [47] has been used to perform simulations for nonspinning binaries with mass ratios $q=1,2,3,4,6,8$ [48-50]. Owing to their high computational complexity, the lengths of these simulations vary between 15 and 33 orbits. Accurate modeling of the late-inspiral and merger phases is important for stellar-mass BBHs, as they merge at frequencies that the advanced detectors would be sensitive to [67]. Analytic models, like those within the EOB formalism, have been calibrated to the NR simulations to increase their accuracy during these phases [51-54]. Other independent models have also been developed using information from NR simulations and their hybrids $[60,61,106,107]$. An alternate derived prescription is that of NR + PN hybrid waveforms, which are constructed by joining long PN early inspirals and late-inspiral merger simulations from NR [55-59].

NR has long sought to contribute template banks for gravitational-wave searches. Because of the restrictions on the length and number of NR waveforms, this has been conventionally pursued by calibrating intermediary waveform models and using those for search templates. In this paper, we explore the alternative of using NR waveforms and their hybrids directly in template banks. We demonstrate the feasibility of this idea for nonspinning binaries, and extending it to spinning binaries would be the subject of a future work. We find that with only six nonspinning NR simulations, we can cover down to $m_{1,2} \gtrsim 12 M_{\odot}$. We show that with 26 additional NR simulations, we can complete the nonspinning template banks down to $M \simeq 12 M_{\odot}$, below which existing PN waveforms have been shown to suffice for aLIGO. From template bank accuracy requirements, we are able to put a bound on the required length and initial GW frequencies for the new simulations. This method can therefore be used to lay down the parameters for future simulations.

First, we construct a bank for using pure-NR waveforms as templates, using a stochastic algorithm similar to Refs. [70-72]. The filter templates are constrained to mass 
ratios for which we have NR simulations available, i.e., $q=1,2,3,4,6,8$. We assume that the simulations available to us are $\geq 20$ orbits in length. To test the bank, we simulate a population of $100,000 \mathrm{BBH}$ signals and filter them through the bank. The signals and templates are both modeled with the EOBNRv2 model [53]. We demonstrate that this bank is indeed effectual and recovers $\geq 97 \%$ of the optimal SNR for GWs from BBHs with mass ratios $1 \leq$ $q \leq 10$ and chirp mass $\mathcal{M}_{c} \equiv\left(m_{1}+m_{2}\right)^{-1 / 5}\left(m_{1} m_{2}\right)^{3 / 5}$ above $27 M_{\odot}$. Figure 3 shows this fraction at different simulated points over the binary mass space. With an additional simulation for $q=9.2$, we are able to extend the coverage to higher mass ratios. We show that a bank viable for NR waveform templates for $q=1,2,3,4,6,9.2$, would recover $\geq 97 \%$ of the optimal SNR for BBHs with $10 \leq q \leq 11$. The SNR recovery fraction from such a bank is shown in Fig. 4.

Second, we construct effectual banks for currently available NR-PN hybrid waveform templates. We derive a bound on waveform model errors, which is independent of analytical models and can be used to independently assess the errors of such models (see Sec. III for details). This allows us to estimate the hybrid waveform mismatches due to PN error, which are negligible at high masses and become significant at lower binary masses. We take their contribution to the SNR loss into account while characterizing template banks. For hybrid banks, we demonstrate and compare two independent algorithms of template bank construction. First, we stochastically place a bank grid, as for the purely NR template bank. Second, we lay down independent sub-banks for each mass ratio, with a fixed overlap between neighboring templates, and take their union as the final bank. To test these banks, we simulate a population of $100,000 \mathrm{BBH}$ signals and filter them through each. We simulate the GW signals and the templates using the recently developed EOBNRv2 model [53]. The fraction of the optimal SNR recovered by the two banks, before and after accounting for the hybrid errors, are shown in the left and right panels of Figs. 6 and 7 (respectively). We observe that for BBHs with $m_{1,2} \geq 12 M_{\odot}$, hybrid template banks will recover $\geq 96.5 \%$ of the optimal SNR. For testing the robustness of our conclusions, we also test the banks using TaylorT4 + NR hybrid templates. The SNR recovery from a bank of these is shown in Fig. 8. We conclude that the currently available NR + PN hybrid waveforms can be used as templates in a matched-filtering search for GWs from BBHs with $m_{1,2} \geq 12 M_{\odot}$ and $1 \leq q \leq 10$. The number of templates required to provide coverage over this region was found to be comparable to a bank constructed using the second-order post-Newtonian TaylorF2 hexagonal template placement method [32,34-36]. The two algorithms we demonstrate yield grids of 667 and 627 templates, respectively, while the metric-based placement method yields a grid of 522 and 736 templates for $97 \%$ and $98 \%$ minimal match, respectively.
At lower mass, the length of the waveform in the sensitive frequency band of the detectors increases, increasing the resolution of the matched filter. We therefore see regions of undercoverage between mass ratios for which we have NR/hybrid templates (see, e.g., Fig. 7 at the left edge). For $M \lesssim 12 M_{\odot}$, existing $\mathrm{PN}$ waveforms were shown to be sufficient for aLIGO searches. We find the additional simulations that would be needed to extend the hybrid template bank down to $12 M_{\odot}$. We show that a bank of hybrids restricted to the 26 mass ratios listed in Table II would be sufficiently dense at $12 M_{\odot}$. This demonstrates that the method proposed here can be used to decide which NR simulations should be prioritized for the purpose of the GW detection problem. By filtering a population of $100,000 \mathrm{BBH}$ signals through this bank, we show that the SNR loss due to its discreteness stays below $2 \%$ over the entire relevant range of masses. The fraction of optimal SNR recovered is shown in Fig. 10. Constraining the detection rate loss below $10 \%$ requires that detection template banks recover more than $96.5 \%$ of the optimal SNR. Therefore, our bank would need hybrids with hybridization mismatches below $1.5 \%$. From this accuracy requirement, we obtain the length requirement for all 26 simulations. This is depicted in the left panel of Fig. 12, where we show the region of the mass space that can be covered with hybrids, as the length of their NR portion varies. We find that for $1 \leq q \leq 10$, the new simulations should be about 50 orbits in length. In the right panel of Fig. 12, we show the corresponding initial GW frequencies. The requirement of $\sim 50$ orbit long NR simulations is ambitious but certainly feasible with the current $\mathrm{BBH}$ simulation technology [68].

In summary, we refer to the right panel of Fig. 10. The region above the dashed (red) line and above the solid (blue) line can be covered with a bank of purely NR waveforms currently available. The region above the dashed (red) and the dashed-dotted (black) line can be covered with the same simulations hybridized to long PN inspirals. With an additional set of NR simulations summarized in Table II, the coverage of the bank can be extended down to the magenta (solid) line in the lower left corner of the figure. Thus, between hybrids and PN waveforms, we can cover the entire nonspinning $\mathrm{BBH}$ space. The ability to use hybrid waveforms within the software infrastructure of the LIGO-Virgo collaboration has been demonstrated by the NINJA-2 collaboration [69]. The template banks we present here can be directly used in aLIGO searches. This work will be most useful when extended to aligned spin and precessing binaries $[108,109]$, which is the subject of a future work.

The detector noise power is modeled using the zerodetuning high-power noise curve for Advanced LIGO [100]. The construction of our template banks is sensitive to the breadth of the frequency range that the detector would be sensitive to. The noise curve we use is the broadband final design sensitivity estimate. For lower sensitivities at the low/high frequencies, our results would 
become more conservative; i.e., the template banks would overcover (and not undercover).

We finally note that in this paper, we have only considered the dominant $(2,2)$ mode of the spherical decomposition of the gravitational waveform. For high mass ratios and high binary masses, other modes would also become important, both for spinning as well as nonspinning black hole binaries $[67,110,111]$. Thus, in future work, it would be relevant to examine the subdominant modes of the gravitational waves. Lastly, though we have looked at the feasibility of using this template bank for Advanced LIGO as a single detector, this instrument will be part of a network of detectors, which comes with increased sensitivity and sky localization. For this reason, in subsequent studies, it would be useful to consider a network of detectors.

\section{ACKNOWLEDGMENTS}

We thank Steve Privitera for useful code contributions and Ian Harry, Alex Nitz, Stefan Ballmer, and the Gravitational-Wave group at Syracuse University for productive discussions. We also thank Mark Hannam and Thomas Dent for carefully reading through the manuscript and providing feedback. D. A. B., P. K., and H. P. P. are grateful for hospitality of the TAPIR group at the California Institute of Technology, where part of this work was completed. D. A. B. and P. K. also thank the LIGO Laboratory Visitors Program, supported by NSF cooperative agreement, Grant No. PHY-0757058, for hospitality during the completion of this work. K. C., I. M., A. H. M., and H.P.P. acknowledge support by NSERC of Canada, the Canada Chairs Program, and the Canadian Institute for Advanced Research. We further acknowledge support from National Science Foundation, Grants No. PHY-0847611 (D. A. B. and P. K.); No. PHY0969111 and No. PHY-1005426 (M. B., L.E. K.); and No. PHY-1068881, No. PHY-1005655, and No. DMS1065438 (M. A. S., B. S., A. Z.). We are grateful for additional support through a Cottrell Scholar award from the Research Corporation for Science Advancement (D. A. B.) and from the Sherman Fairchild Foundation (M. B., L. E. K., M. A. S., B. S., A. Z.). Simulations used in this work were performed with the SpEC code [47]. Calculations were performed on the Zwicky cluster at Caltech, which is supported by the Sherman Fairchild Foundation and by NSF, Grant No. PHY-0960291; on the NSF XSEDE network under Grant No. TG-PHY990007N; on the Syracuse University Gravitation and Relativity cluster, which is supported by NSF, Grants No. PHY-1040231 and No. PHY-1104371 and Syracuse University ITS; and on the GPC supercomputer at the SciNet HPC Consortium [112]. SciNet is funded by the Canada Foundation for Innovation under the auspices of Compute Canada, the Government of Ontario, Ontario Research Fund-Research Excellence, and the University of Toronto.
[1] G. M. Harry et al. (LIGO Scientific Collaboration), Classical Quantum Gravity 27, 084006 (2010).

[2] F. Acernese et al. (The Virgo Collaboration), VIR-0027A092009.

[3] J. Aasi et al. (LIGO Scientific Collaboration, Virgo Collaboration), arXiv:1304.0670.

[4] K. Somiya (KAGRA Collaboration), Classical Quantum Gravity 29, 124007 (2012).

[5] J. Abadie et al. (LIGO Scientific Collaboration, Virgo Collaboration), Classical Quantum Gravity 27, 173001 (2010).

[6] A. Tutukov and L. Yungelson, Nauchnye Informatsii 27, 86 (1973).

[7] A. Tutukov and L. Yungelson, Nauchnye Informatsii 27, 70 (1973).

[8] R. Webbink, Astrophys. J. 277, 355 (1984).

[9] I. Kowalska, T. Regimbau, T. Bulik, M. Dominik, and K. Belczynski, arXiv:1205.4621.

[10] C. L. Fryer, K. Belczynski, G. Wiktorowicz, M. Dominik, V. Kalogera, and D. E. Holz, Astrophys. J. 749, 91 (2012).

[11] M. Dominik, K. Belczynski, C. Fryer, D. E. Holz, E. Berti, T. Bulik, I. Mandel, and R. O'Shaughnessy, Astrophys. J. 759, 52 (2012).

[12] K. Belczynski and M. Dominik, arXiv:1208.0358.
[13] J. Abadie et al. (LIGO Collaboration, Virgo Collaboration), Phys. Rev. D 81, 102001 (2010).

[14] J. Abadie et al. (Virgo Collaboration), Astrophys. J. 734, L35 (2011).

[15] J. Abadie et al. (LIGO Scientific Collaboration, Virgo Collaboration), Phys. Rev. D 85, 122007 (2012).

[16] J. Abadie et al. (LIGO Scientific Collaboration, Virgo Collaboration), Phys. Rev. D 85, 082002 (2012).

[17] J. Abadie et al. (LIGO Scientific Collaboration, Virgo Collaboration), Phys. Rev. D 82, 102001 (2010).

[18] B. Abbott et al. (LIGO Scientific Collaboration), Phys. Rev. D 80, 047101 (2009).

[19] B. Abbott et al. (LIGO Scientific Collaboration), Phys. Rev. D 79, 122001 (2009).

[20] E. Messaritaki (LIGO Scientific Collaboration), Classical Quantum Gravity 22, S1119 (2005).

[21] J. Abadie et al. (LIGO Scientific Collaboration, Virgo Collaboration), Phys. Rev. D 83, 122005 (2011).

[22] J. Aasi et al. (LIGO Scientific Collaboration, Virgo Collaboration), Phys. Rev. D 87, 022002 (2013).

[23] B. Abbott et al. (LIGO Scientific Collaboration), Phys. Rev. D 80, 062001 (2009).

[24] B. Abbott et al. (LIGO Scientific Collaboration), Phys. Rev. D 69, 082004 (2004). 
[25] B. Abbott et al. (LIGO Scientific Collaboration), Phys. Rev. D 72, 102004 (2005).

[26] A. M. Sintes (LIGO Scientific Collaboration), J. Phys. Conf. Ser. 39, 36 (2006).

[27] J. Abadie et al. (LIGO Scientific Collaboration, Virgo Collaboration), Astrophys. J. 737, 93 (2011).

[28] C. Palomba (LIGO Scientific Collaboration, Virgo Collaboration), arXiv:1201.3176.

[29] L. A. Wainstein and V. D. Zubakov, Extraction of Signals from Noise (Prentice-Hall, Englewood Cliffs, NJ, 1962).

[30] B. Allen, W. G. Anderson, P. R. Brady, D. A. Brown, and J. D. E. Creighton, Phys. Rev. D 85, 122006 (2012).

[31] B. S. Sathyaprakash and S. V. Dhurandhar, Phys. Rev. D 44, 3819 (1991).

[32] B. J. Owen and B. S. Sathyaprakash, Phys. Rev. D 60, 022002 (1999).

[33] B. J. Owen, Phys. Rev. D 53, 6749 (1996).

[34] S. Babak, R. Balasubramanian, D. Churches, T. Cokelaer, and B. Sathyaprakash, Classical Quantum Gravity 23, 5477 (2006).

[35] B. S. Sathyaprakash, Phys. Rev. D 50, R7111 (1994).

[36] T. Cokelaer, Phys. Rev. D 76, 102004 (2007).

[37] G. L. Turin, IRE Trans. Inf. Theory 6, 311 (1960).

[38] F. Pretorius, Phys. Rev. Lett. 95, 121101 (2005).

[39] J. G. Baker, J. Centrella, D.-I. Choi, M. Koppitz, and J. van Meter, Phys. Rev. Lett. 96, 111102 (2006).

[40] M. Campanelli, C. O. Lousto, P. Marronetti, and Y. Zlochower, Phys. Rev. Lett. 96, 111101 (2006).

[41] F. Pretorius, Classical Quantum Gravity 23, S529 (2006).

[42] L. Lindblom, M. A. Scheel, L. E. Kidder, R. Owen, and O. Rinne, Classical Quantum Gravity 23, S447 (2006).

[43] F. Pretorius, arXiv:0710.1338.

[44] M. Hannam, Classical Quantum Gravity 26, 114001 (2009).

[45] I. Hinder, Classical Quantum Gravity 27, 114004 (2010).

[46] H. P. Pfeiffer, Classical Quantum Gravity 29, 124004 (2012).

[47] http://www.black-holes.org/SpEC.html.

[48] L. T. Buchman, H. P. Pfeiffer, M. A. Scheel, and B. Szilágyi, Phys. Rev. D 86, 084033 (2012).

[49] A. H. Mroué and H. P. Pfeiffer, arXiv:1210.2958.

[50] A. H. Mroué et al., Phys. Rev. Lett. 111, 241104 (2013).

[51] A. Buonanno and T. Damour, Phys. Rev. D 59, 084006 (1999).

[52] A. Buonanno, Yi Pan, H. P. Pfeiffer, M. Scheel, L. Buchman, and L. Kidder, Phys. Rev. D 79, 124028 (2009).

[53] Y. Pan, A. Buonanno, M. Boyle, L. T. Buchman, L. E. Kidder, H. P. Pfeiffer, and M. A. Scheel, Phys. Rev. D 84, 124052 (2011).

[54] A. Taracchini, Y. Pan, A. Buonanno, E. Barausse, M. Boyle, T. Chu, G. Lovelace, H. P. Pfeiffer, and M. A. Scheel, Phys. Rev. D 86, 024011 (2012).

[55] M. Boyle, Phys. Rev. D 84, 064013 (2011).

[56] I. MacDonald, S. Nissanke, and H. P. Pfeiffer, Classical Quantum Gravity 28, 134002 (2011).

[57] I. MacDonald, A. H. Mroué, H. P. Pfeiffer, M. Boyle, L. E. Kidder, M. A. Scheel, B. Szilágyi, and N. W. Taylor, Phys. Rev. D 87, 024009 (2013).

[58] F. Ohme, M. Hannam, and S. Husa, Phys. Rev. D 84, 064029 (2011).
[59] M. Hannam, S. Husa, F. Ohme, and P. Ajith, Phys. Rev. D 82, 124052 (2010).

[60] P. Ajith et al., Classical Quantum Gravity 24, S689 (2007).

[61] L. Santamaria et al., Phys. Rev. D 82, 064016 (2010).

[62] L. Cadonati et al., Classical Quantum Gravity 26, 114008 (2009).

[63] P. Ajith et al., Classical Quantum Gravity 29, 124001 (2012).

[64] L. Santamaria, B. Krishnan, and J. T. Whelan, Classical Quantum Gravity 26, 114010 (2009).

[65] B. Aylott et al., Classical Quantum Gravity 26, 165008 (2009).

[66] A. Buonanno, B. R. Iyer, E. Ochsner, Y. Pan, and B. S. Sathyaprakash, Phys. Rev. D 80, 084043 (2009).

[67] D. A. Brown, P. Kumar, and A. H. Nitz, Phys. Rev. D 87, 082004 (2013).

[68] B. Szilágyi, VIR-0027A-09 http://www.grg.uib.es/ NRDA13/slides/Szilagyi_StretchingTheLimitsOfNR.pdf.

[69] J. Abadie et al. (NINJA-2 Collaboration, LIGO Collaboration, and Virgo Collaboration), arXiv:1401.0939.

[70] I. W. Harry, B. Allen, and B. S. Sathyaprakash, Phys. Rev. D 80, 104014 (2009).

[71] P. Ajith, N. Fotopoulos, S. Privitera, A. Neunzert, and A. J. Weinstein, arXiv:1210.6666.

[72] G. M. Manca and M. Vallisneri, Phys. Rev. D 81, 024004 (2010).

[73] T. A. Apostolatos, Phys. Rev. D 52, 605 (1995).

[74] B. S. Sathyaprakash, Classical Quantum Gravity 17, L157 (2000).

[75] M. A. Scheel, M. Boyle, T. Chu, L. E. Kidder, K. D. Matthews, and H. P. Pfeiffer, Phys. Rev. D 79, 024003 (2009).

[76] M. Boyle, D. A. Brown, L. E. Kidder, A. H. Mroué, H. P. Pfeiffer, M. A. Scheel, G. B. Cook, and S. A. Teukolsky, Phys. Rev. D 76, 124038 (2007).

[77] P. Jaranowski and G. Schäfer, Phys. Rev. D 57, 7274 (1998).

[78] P. Jaranowski and G. Schäfer, Phys. Rev. D 60, 124003 (1999).

[79] P. Jaranowski and G. Schaefer, Ann. Phys. (N.Y.) 9, 378 (2000).

[80] T. Damour, P. Jaranowski, and G. Schaefer, Phys. Lett. B 513, 147 (2001).

[81] L. Blanchet, T. Damour, and G. Esposito-Farèse, Phys. Rev. D 69, 124007 (2004).

[82] T. Damour, P. Jaranowski, and G. Schäfer, Phys. Rev. D 63, 044021 (2001).

[83] L. Blanchet and B. R. Iyer, Classical Quantum Gravity 20, 755 (2003).

[84] L. Blanchet, G. Faye, B. R. Iyer, and B. Joguet, Phys. Rev. D 65, 061501 (2002).

[85] L. Blanchet, T. Damour, G. Esposito-Farèse, and B. R. Iyer, Phys. Rev. Lett. 93, 091101 (2004).

[86] L. Blanchet, T. Damour, G. Esposito-Farèse, and B. R. Iyer, Phys. Rev. D 71, 124004 (2005).

[87] L. Blanchet and B. R. Iyer, Phys. Rev. D 71, 024004 (2005).

[88] J. Mathews and R. L. Walker, Mathematical Methods of Physics (Benjamin, New York, 1970), 2nd ed.

[89] L. Blanchet, Living Rev. Relativity 9, 4 (2006). 
[90] J. D. Creighton and W. G. Anderson, Gravitational-Wave Physics and Astronomy: An Introduction to Theory, Experiment and Data Analysis (Wiley, New York, 2011).

[91] A. Buonanno and T. Damour, Phys. Rev. D 62, 064015 (2000).

[92] T. Damour, B. R. Iyer, P. Jaranowski, and B. S. Sathyaprakash, Phys. Rev. D 67, 064028 (2003).

[93] T. Damour and A. Nagar, Phys. Rev. D 77, 024043 (2008).

[94] T. Damour, B. R. Iyer, and A. Nagar, Phys. Rev. D 79, 064004 (2009).

[95] T. Damour, P. Jaranowski, and G. Schäfer, Phys. Rev. D 62, 084011 (2000).

[96] T. Damour and A. Nagar, Phys. Rev. D 79, 081503 (2009).

[97] T. Damour, A. Nagar, E. N. Dorband, D. Pollney, and L. Rezzolla, Phys. Rev. D 77, 084017 (2008).

[98] E. Berti, V. Cardoso, and C. M. Will, Phys. Rev. D 73, 064030 (2006).

[99] R. Balasubramanian, B. S. Sathyaprakash, and S. V. Dhurandhar, Phys. Rev. D 53, 3033 (1996).

[100] LIGO(David Shoemaker), Technical Report No. T0900288v3, 2009.

[101] L. Lindblom, B. J. Owen, and D. A. Brown, Phys. Rev. D 78, 124020 (2008).
[102] L. Lindblom, J. G. Baker, and B. J. Owen, Phys. Rev. D 82, 084020 (2010).

[103] K. Cannon, J. D. Emberson, C. Hanna, D. Keppel, and H. P. Pfeiffer, Phys. Rev. D 87, 044008 (2013).

[104] M. A. Scheel, B. Szilágyi, and H. P. Pfeiffer (private communication).

[105] S. Babak et al., Phys. Rev. D 87, 024033 (2013).

[106] Numerical Relativity-Analytical Relativity Collaboration (NRAR).

[107] E. A. Huerta, P. Kumar, and D. A. Brown, Phys. Rev. D 86, 024024 (2012).

[108] M. Boyle, Phys. Rev. D 87, 104006 (2013).

[109] P. Schmidt, M. Hannam, and S. Husa, Phys. Rev. D 86, 104063 (2012).

[110] L. Pekowsky, J. Healy, D. Shoemaker, and P. Laguna, Phys. Rev. D 87, 084008 (2013).

[111] C. Capano, Y. Pan, and A. Buonanno, arXiv:1311.1286.

[112] C. Loken, D. Gruner, L. Groer, R. Peltier, N. Bunn, M. Craig, T. Henriques, J. Dempsey, C.-H. Yu, J. Chen, L. J. Dursi, J. Chong, S. Northrup, J. Pinto, N. Knecht, and R. V. Zon, J. Phys. Conf. Ser. 256, 012026 (2010). 